\title{
Strategy for Contiguous Tetramination of Cyclohexanones with $o$-Phenylenediamines with Elemental Sulfur and DMSO
}

\author{
Thanh Binh Nguyen, ${ }^{* \dagger \dagger}$ Le Anh Nguyen, ${ }^{\dagger, \S}$ and Pascal Retailleau ${ }^{\dagger}$ \\ ${ }^{\dagger}$ Institut de Chimie des Substances Naturelles, CNRS UPR 2301, Université Paris-Sud, Université Paris-Saclay, \\ 1 avenue de la Terrasse, 91198 Gif-sur-Yvette, France \\ \$Institute of Chemistry, Vietnam Academy of Science and Technology, 18 Hoang Quoc Viet, Cau Giay, Hanoi, \\ Vietnam \\ ${ }^{\S}$ Graduate University of Science and Technology, 18 Hoang Quoc Viet, Cau Giay, Hanoi, Vietnam
}

\section{General information}

Reagents were obtained from commercial supplier and used without further purification. Analytical thin layer chromatography (TLC) was purchased from Merck KGaA (silica gel 60 F254). Visualization of the chromatogram was performed by UV light $(254 \mathrm{~nm})$ or phosphomolybdic acid or vanilline stains. Flash column chromatography was carried out using kieselgel 35-70 $\mu \mathrm{m}$ particle sized silica gel (230-400 mesh). NMR Chemical shifts are reported in $(\delta)$ ppm relative to tetramethylsilane (TMS) with the residual solvent as internal reference $\left(\mathrm{CDCl}_{3}, \delta 7.26 \mathrm{ppm}\right.$ for ${ }^{l} \mathrm{H}$ and $\delta 77.0 \mathrm{ppm}$ for ${ }^{13} \mathrm{C}$; DMSO- $d_{6}, \delta 2.50 \mathrm{ppm}$ for ${ }^{1} \mathrm{H}$ and $\delta 39.5 \mathrm{ppm}$ for ${ }^{13} \mathrm{C}$; $\mathrm{CD}_{3} \mathrm{OD}, \delta 3.31 \mathrm{ppm}$ for ${ }^{1} \mathrm{H}$ and $\delta 49.0 \mathrm{ppm}$ for ${ }^{13} \mathrm{C}$ ). Data are reported as follows: chemical shift, multiplicity $(\mathrm{s}=$ singlet, $\mathrm{d}=$ doublet, $\mathrm{t}=$ triplet, $\mathrm{q}$ $=$ quartet, $\mathrm{m}=$ multiplet $)$, coupling constants $(\mathrm{Hz})$ and integration.

\section{General procedure (Applied for Table 1, entry 10; Tables 2 and 3; Scheme 3)}

A mixture of cyclohexanone 1 ( $1 \mathrm{mmol})$, elemental sulfur $(5 \mathrm{mmol}, 160 \mathrm{mg})$ and TFA $(0.2 \mathrm{mmol}, 23$ $\mathrm{mg}$ ), and DMSO (1 mL, $15 \mathrm{mmol})$ was heated under an argon atmosphere in a 7-mL test tube at $100{ }^{\circ} \mathrm{C}$ for $16 \mathrm{~h}$. The reaction mixture was diluted with methanol $(2 \mathrm{~mL})$. The resulting mixture was diluted with $\mathrm{CH}_{2} \mathrm{Cl}_{2}$. The resulting mixture was washed with saturated aqueous $\mathrm{NaHCO}_{3}$ solution. The organic layer separated was dried $\left(\mathrm{Na}_{2} \mathrm{SO}_{4}\right)$ and concentrated. The residue was purified by washing with methanol or by column chromatography on silica gel $\left(\mathrm{CH}_{2} \mathrm{Cl}_{2}\right.$ to $\mathrm{CH}_{2} \mathrm{Cl}_{2}$ :EtOAc 9:1 to 8:2). In case of 3aj, the reaction mixture was triturated with $\mathrm{MeOH}$ and filtered. The residue was washed with $\mathrm{MeOH}$ and dried to afford the product as a yellow solid.

The reactions described in Schemes 2 and 4 were carried out in the same manner with modification of the amount of chemicals. 


\section{Characterizations of products}

$N^{1}$-(phenazin-1-yl)benzene-1,2-diamine (4)<smiles>Nc1ccccc1Nc1cnc2ccccc2n1</smiles>

Red solid (186 mg, 65\% for Table 1, entry 1; $200 \mathrm{mg}, 70 \%$ for Table 1, entry 7).

${ }^{1} \mathrm{H}$ NMR $\left(300 \mathrm{MHz}, \mathrm{CDCl}_{3}\right) \delta$ 8.25-8.17 (m, 2H), 7.86-7.75 (m, 3H), 7.65-7.55 (m, 2H), $7.36(\mathrm{~d}, J=$ $8.0 \mathrm{~Hz}, 1 \mathrm{H}), 7.16(\mathrm{t}, J=8.0 \mathrm{~Hz}, 1 \mathrm{H}), 6.93-6.84(\mathrm{~m}, 2 \mathrm{H}), 6.74(\mathrm{~d}, J=8.0 \mathrm{~Hz}, 1 \mathrm{H}), 3.91$ (broad s, 2H).

${ }^{13} \mathrm{C}$ NMR $\left(75 \mathrm{MHz}, \mathrm{CDCl}_{3}\right) \delta 144.3,143.9,143.1,142.2,141.0,135.2,132.5,130.6,129.7$ (2C), $129.5,127.1,126.7,119.2,117.0,116.4,105.6$ (1 signal missing due to overlap).

HRMS (ESI+) calcd for $\mathrm{C}_{18} \mathrm{H}_{15} \mathrm{~N}_{4}[\mathrm{M}+\mathrm{H}]^{+}$287.1297. Found 287.1304.

\section{Quinoxalino[2,3-a]phenazine (3aa) ${ }^{1}$}<smiles>c1ccc2nc3c(ccc4nc5ccccc5nc43)nc2c1</smiles>

Pale yellow solid (220 mg, 78\% for Table 1, entry 10).

${ }^{1} \mathrm{H}$ NMR $\left(300 \mathrm{MHz}, \mathrm{CDCl}_{3}\right) \delta$ 8.66-8.60 (m, 2H), 8.32-8.26 (m, 2H), 8.21 (s, 2H), 7.97-7.91 (m, 4H).

${ }^{13} \mathrm{C}$ NMR $\left(75 \mathrm{MHz}, \mathrm{CDCl}_{3}\right) \delta 145.5,143.4,142.4,142.3,133.5,131.8,131.0,130.9,129.5$.

HRMS (ESI+) calcd for $\mathrm{C}_{18} \mathrm{H}_{11} \mathrm{~N}_{4}[\mathrm{M}+\mathrm{H}]^{+}$283.0984. Found 283.0965 .

6-Methylquinoxalino[2,3-a]phenazine (3ab)<smiles>Cc1cc2nc3ccccc3nc2c2nc3ccccc3nc12</smiles>

Pale yellow solid (231 mg, 78\% from 4-methylcyclohexanone; $222 \mathrm{mg}$, 75\% from 3methylcyclohexanone; $74 \mathrm{mg}$, 25\% from 2-methylcyclohexanone for Table 2, entries 1-3 respectively). ${ }^{1} \mathrm{H}$ NMR $\left(300 \mathrm{MHz}, \mathrm{CDCl}_{3}\right) \delta 8.66-8.62(\mathrm{~m}, 2 \mathrm{H}), 8.38-8.37(\mathrm{~m}, 1 \mathrm{H}), 8.31-8.29(\mathrm{~m}, 1 \mathrm{H}), 8.11(\mathrm{~s}, 1 \mathrm{H})$, 7.97-7.89 (m, 4H), 2.97 (s, 3H).

${ }^{13} \mathrm{C}$ NMR (75 MHz, $\left.\mathrm{CDCl}_{3}\right) \delta 145.7(2 \mathrm{C}), 143.5,143.0,142.2$ (2C), 142.1, 141.9, 141.8, 131.5, 131.4, $131.3,130.9,130.8,130.7,130.4,129.8,129.2,18.8$.

HRMS (ESI+) calcd for $\mathrm{C}_{19} \mathrm{H}_{13} \mathrm{~N}_{4}[\mathrm{M}+\mathrm{H}]^{+}$297.1140. Found 297.1147 .

\footnotetext{
${ }^{1}$ Albini, A.; Bettinetti, G. F.; Minoli, G. J. Chem. Soc. Perkin Trans. 1 1981, 1821.
} 
<smiles></smiles>

Pale yellow solid (259 mg, 80\% from 4-propylcyclohexanone, Table 2, entry 4).

${ }^{1} \mathrm{H}$ NMR $\left(300 \mathrm{MHz}, \mathrm{CDCl}_{3}\right) \delta 8.53-8.48(\mathrm{~m}, 2 \mathrm{H}), 8.22-8.14(\mathrm{~m}, 2 \mathrm{H}), 7.90(\mathrm{~s}, 1 \mathrm{H}), 7.84-7.76(\mathrm{~m}, 2 \mathrm{H})$, 3.25 (t, $J=7.4 \mathrm{~Hz}, 2 \mathrm{H}), 1.89$ (sextet, $J=7.4 \mathrm{~Hz}, 2 \mathrm{H}$ ), 1.07 (t, $J=7.4 \mathrm{~Hz}, 3 \mathrm{H}$ ).

${ }^{13} \mathrm{C}$ NMR $\left(75 \mathrm{MHz}, \mathrm{CDCl}_{3}\right) \delta 145.9,145.6,145.1,143.4,142.8,142.1,142.0,141.9,141.7,131.4$, $131.2,130.8,130.7,130.6,130.2(2 \mathrm{C}), 129.8,129.1,33.5,22.3,14.3$.

HRMS (ESI+) calcd for $\mathrm{C}_{21} \mathrm{H}_{17} \mathrm{~N}_{4}[\mathrm{M}+\mathrm{H}]^{+}$325.1453. Found 325.1436 .

\section{6-Pentylquinoxalino[2,3-a]phenazine (3ad)}<smiles>CCCCCc1cc2nc3ccccc3nc2c2nc3ccccc3nc12</smiles>

Pale yellow solid (285 mg, 81\% from 4-pentylcyclohexanone, Table 2, entry 5).

${ }^{1} \mathrm{H}$ NMR (300 MHz, $\left.\mathrm{CDCl}_{3}\right) \delta 8.60-8.53(\mathrm{~m}, 2 \mathrm{H}), 8.31-8.20(\mathrm{~m}, 2 \mathrm{H}), 7.99(\mathrm{~s}, 1 \mathrm{H}), 7.90-7.81(\mathrm{~m}, 4 \mathrm{H})$, 3.33 (t, $J=7.4 \mathrm{~Hz}, 2 \mathrm{H}), 1.91$ (quintet, $J=7.4 \mathrm{~Hz}, 2 \mathrm{H}$ ), 1.55-1.36 (m, 4H), 0.91 (t, $J=7.4 \mathrm{~Hz}, 2 \mathrm{H}$ ).

${ }^{13} \mathrm{C}$ NMR $\left(75 \mathrm{MHz}, \mathrm{CDCl}_{3}\right) \delta 146.4,145.8,145.3,143.5,142.9,142.2$ (2C), 142.0, 141.9, 131.5, $131.4,130.9,130.8,130.7,130.4,130.3,129.9,129.2,32.0,31.5,28.9,22.8,14.2$.

HRMS (ESI+) calcd for $\mathrm{C}_{23} \mathrm{H}_{21} \mathrm{~N}_{4}[\mathrm{M}+\mathrm{H}]^{+}$353.1766. Found 353.1780.

\section{6-Isopropylquinoxalino[2,3-a]phenazine (3ae)}<smiles></smiles>

Pale yellow solid (243 mg, 75\% from 4-isopropylcyclohexanone; $249 \mathrm{mg}$, $77 \mathrm{mg}$ from 3isopropylcyclohexanone; Table 2, entries 6 and 7, respectively).

${ }^{1} \mathrm{H}$ NMR (300 MHz, $\left.\mathrm{CDCl}_{3}\right) \delta$ 8.61-8.55 (m, 2H), 8.32-8.22 (m, 2H), 8.07 (s, 1H), 7.92-7.83 (m, 4H), 4.35 (septet, $J=6.9 \mathrm{~Hz}, 1 \mathrm{H}), 1.51(\mathrm{~d}, J=6.9 \mathrm{~Hz}, 6 \mathrm{H})$.

${ }^{13} \mathrm{C}$ NMR $\left(75 \mathrm{MHz}, \mathrm{CDCl}_{3}\right) \delta 152.0,145.9,144.7,143.5,142.8,142.3,142.1,142.0,141.8,131.5$, $131.4,130.9,130.8,130.7,130.4,129.9,129.2,127.8,28.0,22.9$.

HRMS (ESI+) calcd for $\mathrm{C}_{21} \mathrm{H}_{17} \mathrm{~N}_{4}[\mathrm{M}+\mathrm{H}]^{+}$325.1453. Found 325.1431 . 
<smiles>CC(C)(C)c1cc2nc3ccccc3nc2nc1-c1ccccc1</smiles>

Pale yellow solid (237 mg, 70\% from 4-tert-butylcyclohexanone; $243 \mathrm{mg}$, 72\% from 3-tertbutylcyclohexanone; Table 2, entries 8 and 9 , respectively).

${ }^{1} \mathrm{H}$ NMR $\left(300 \mathrm{MHz}, \mathrm{CDCl}_{3}\right) \delta$ 8.63-8.59 (m, 2H), 8.34-8.26 (m, 2H), $8.18(\mathrm{~s}, 1 \mathrm{H}), 7.93-7.85(\mathrm{~m}, 4 \mathrm{H})$, $1.81(\mathrm{~s}, 9 \mathrm{H})$.

${ }^{13} \mathrm{C}$ NMR $\left(75 \mathrm{MHz}, \mathrm{CDCl}_{3}\right) \delta 152.7,145.7,143.8,142.7,142.4,142.3,141.8,141.0,131.6,131.3$, $131.0,130.9,130.6,130.5,129.9,129.3,129.2,37.6,30.9$ (1 signal missing due to overlap).

HRMS (ESI+) calcd for $\mathrm{C}_{22} \mathrm{H}_{19} \mathrm{~N}_{4}[\mathrm{M}+\mathrm{H}]^{+} 339.1610$. Found 339.1632.

6-Phenylquinoxalino $[2,3-a]$ phenazine (3ag)<smiles>c1ccc(-c2cc3nc4ccccc4nc3c3nc4ccccc4nc23)cc1</smiles>

Pale yellow solid (261 mg, 73\% from 4-phenylcyclohexanone, Table 2, entry 10).

${ }^{1} \mathrm{H}$ NMR $\left(300 \mathrm{MHz}, \mathrm{CDCl}_{3}\right) \delta$ 8.67-8.64 (m, 2H), 8.34-8.27 (m, 3H), 7.98-7.88 (m, 6H), 7.62-7.52 (m, $3 \mathrm{H})$.

${ }^{13} \mathrm{C}$ NMR $\left(75 \mathrm{MHz}, \mathrm{CDCl}_{3}\right) \delta 145.5,144.7,144.5,144.0,143.0,142.5,142.4,141.9,137.3,132.7$, $131.9,131.6,131.4,31.0(2 \mathrm{C}), 130.9,130.7,130.2,129.5,129.0,128.5$ (1 signal missing due to overlap).

HRMS (ESI+) calcd for $\mathrm{C}_{24} \mathrm{H}_{15} \mathrm{~N}_{4}[\mathrm{M}+\mathrm{H}]^{+}$359.1297. Found 359.1318.

6-Benzylquinoxalino[2,3-a]phenazine (3ah)<smiles>c1ccc(Cc2cc3nc4ccccc4nc3nc2-c2ccccc2)cc1</smiles>

Pale yellow solid (242 mg, 65\% from 2-benzylcyclohexanone, Table 2, entry 11).

${ }^{1} \mathrm{H}$ NMR $\left(300 \mathrm{MHz}, \mathrm{CDCl}_{3}\right) \delta 8.69-8.60(\mathrm{~m}, 2 \mathrm{H}), 8.42-8.37(\mathrm{~m}, 1 \mathrm{H}), 8.28-8.22(\mathrm{~m}, 1 \mathrm{H}), 7.99-7.88(\mathrm{~m}$, $5 \mathrm{H}), 7.52-7.49(\mathrm{~m}, 2 \mathrm{H}), 7.38-7.32(\mathrm{~m}, 2 \mathrm{H}), 7.28-7.23(\mathrm{~m}, 1 \mathrm{H}), 4.79(\mathrm{~s}, 2 \mathrm{H})$.

${ }^{13} \mathrm{C}$ NMR $\left(75 \mathrm{MHz}, \mathrm{CDCl}_{3}\right) \delta 145.8,145.3,145.1,143.6,143.0,142.4,142.3,142.2,142.1,139.2$, 131.7, 131.6 (2C), 131.1, 131.0, 130.8, 130.7, 130.1, 130.0, 129.3, 128.9, 126.8, 37.6.

HRMS (ESI+) calcd for $\mathrm{C}_{25} \mathrm{H}_{17} \mathrm{~N}_{4}[\mathrm{M}+\mathrm{H}]^{+}$373.1453. Found 373.1481 . 
Ethyl quinoxalino[2,3-a]phenazine-6-carboxylate (3ai)<smiles></smiles>

Pale yellow solid (237 mg, 67\% from 4-ethoxycarbonylcyclohexanone, Table 2, entry 12).

${ }^{1} \mathrm{H}$ NMR (300 MHz, $\left.\mathrm{CDCl}_{3}\right) \delta 8.64-8.58(\mathrm{~m}, 2 \mathrm{H}), 8.53(\mathrm{~s}, 1 \mathrm{H}), 8.36-8.28(\mathrm{~m}, 2 \mathrm{H}), 7.97-7.89(\mathrm{~m}, 4 \mathrm{H})$, $4.62(\mathrm{q}, J=7.1 \mathrm{~Hz}, 2 \mathrm{H}), 1.51(\mathrm{t}, J=7.1 \mathrm{~Hz}, 3 \mathrm{H})$.

${ }^{13} \mathrm{C}$ NMR $\left(75 \mathrm{MHz}, \mathrm{CDCl}_{3}\right) \delta 166.0,144.2,143.8,143.1,142.9,142.8,142.7,142.2,142.0,136.1$, $134.0,132.1,131.9,131.8,131.7,130.9,130.7,130.2,129.7,62.3,14.6$.

HRMS (ESI+) calcd for $\mathrm{C}_{21} \mathrm{H}_{15} \mathrm{~N}_{4} \mathrm{O}_{2}[\mathrm{M}+\mathrm{H}]^{+}$355.1195. Found 355.1182.

$N$-(Quinoxalino[2,3-a]phenazin-6-yl)acetamido (3aj)<smiles></smiles>

The crude mixture was triturated with methanol, filtered and washed with methano to provide the product as a yellow solid (237 mg, 70\% from 4-pentylcyclohexanone, Table 2, entry 13).

${ }^{1} \mathrm{H}$ NMR (300 MHz, $\left.\mathrm{CDCl}_{3}\right) \delta 9.79$ (broad s, $\left.1 \mathrm{H}\right), 9.19(\mathrm{~s}, 1 \mathrm{H}), 8.66-8.60(\mathrm{~m}, 1 \mathrm{H}), 8.54-8.51(\mathrm{~m} \mathrm{1H})$, 8.32-8.22 (m, 2H), 7.98-7.81 (m, 4H), $2.46(\mathrm{~s}, 3 \mathrm{H})$.

${ }^{13} \mathrm{C}$ NMR $\left(75 \mathrm{MHz}, \mathrm{CDCl}_{3}\right) \delta 169.4,147.0,144.0,142.8,142.1,141.6,141.3,140.2,138.5,135.7$, $132.3,131.8,131.4,131.0,130.8,130.2,129.3,129.0,116.7,25.5$.

HRMS (ESI+) calcd for $\mathrm{C}_{20} \mathrm{H}_{14} \mathrm{~N}_{5} \mathrm{O}[\mathrm{M}+\mathrm{H}]^{+} 340.1198$. Found 340.1181 .

7,8-Dihydro-6H-cyclohepta[1,2-b:3,4-b']diquinoxaline (3ak)<smiles></smiles>

Pale yellow solid (89 mg, 30\%, Scheme 2).

${ }^{1} \mathrm{H}$ NMR $\left(300 \mathrm{MHz}, \mathrm{CDCl}_{3}\right) \delta 8.33-8.30(\mathrm{~m} \mathrm{2H}), 8.12-8.08(\mathrm{~m} \mathrm{2H}), 7.82-7.73(\mathrm{~m}, 4 \mathrm{H}), 3.06(\mathrm{t}, J=7.4$ $\mathrm{Hz}, 4 \mathrm{H}$ ), 2.51 (quintet, $J=7.4 \mathrm{~Hz}, 2 \mathrm{H}$ ).

${ }^{13} \mathrm{C} \mathrm{NMR}\left(75 \mathrm{MHz}, \mathrm{CDCl}_{3}\right) \delta 154.9,151.8,142.5,142.2,131.0,130.4,130.4,130.0,128.8,33.6,28.4$. HRMS (ESI+) calcd for $\mathrm{C}_{19} \mathrm{H}_{15} \mathrm{~N}_{4}[\mathrm{M}+\mathrm{H}]^{+}$299.1297. Found 299.1312 . 


\section{2,3,11,12-Tetramethyl-7,8-dihydro-6H-cyclohepta[1,2-b:3,4-b']diquinoxaline (3bk)}

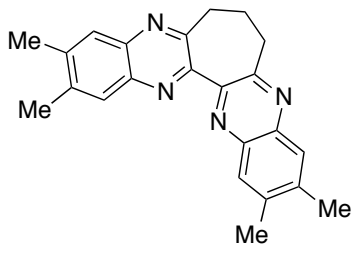

Pale yellow solid (124 mg, 35\%, Scheme 2).

${ }^{1} \mathrm{H}$ NMR (300 MHz, CDCl $) \delta 8.04(\mathrm{~s}, 2 \mathrm{H}), 7.83(\mathrm{~s}, 2 \mathrm{H}), 3.00(\mathrm{t}, J=7.2 \mathrm{~Hz}, 4 \mathrm{H}), 2.53-2.40(\mathrm{~m}, 14 \mathrm{H})$.

${ }^{13} \mathrm{C}$ NMR $\left(75 \mathrm{MHz}, \mathrm{CDCl}_{3}\right) \delta 16$.

HRMS (ESI + ) calcd for $\mathrm{C}_{23} \mathrm{H}_{23} \mathrm{~N}_{4}[\mathrm{M}+\mathrm{H}]^{+}$355.1923. Found 355.1902.

6,6-Dimethyl-6,7-dihydroquinoxalino[2,3-a]phenazine (3al)<smiles>CC1(C)Cc2nc3ccccc3nc2Nc2nc3ccccc3nc21</smiles>

Pale yellow solid (203 mg, 65\% from 4,4-dimethylcyclohexanone; $215 \mathrm{mg}$, 69\% from 3,3dimethylcyclohexanone; Table 3, entries 1 and 2, respectively).

${ }^{1} \mathrm{H}$ NMR (300 MHz, $\left.\mathrm{CDCl}_{3}\right) \delta 8.42-8.37$ (m, 2H), 8.12-8.05 (m, 2H), 7.80-7.73 (m, 4H), $3.48(\mathrm{~s}, 2 \mathrm{H})$, $1.54(\mathrm{~s}, 6 \mathrm{H})$.

${ }^{13} \mathrm{C}$ NMR $\left(75 \mathrm{MHz}, \mathrm{CDCl}_{3}\right) \delta 161.0,154.0,146.3,145.1,142.9$ (2C), 142.7, 142.3, 131.2, 131.1, $130.8,130.6,130.0(2 \mathrm{C}), 129.2,128.7,46.0,38.4,27.7$.

HRMS (ESI+) calcd for $313.1453[\mathrm{M}+\mathrm{H}]^{+} 313.1453$. Found 313.1462.

\section{2,3,6,6,10,11-Hexamethyl-6,7-dihydroquinoxalino[2,3-a]phenazine (3bl)}

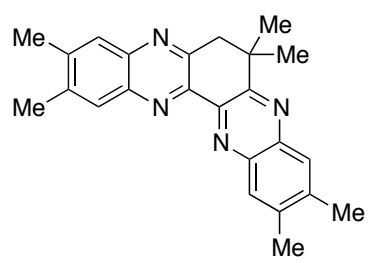

Pale yellow solid (232 mg, 63\% from 4,4-dimethylcyclohexanone; $258 \mathrm{mg}, 70 \%$ from 3,3dimethylcyclohexanone; Table 3, entries 3 and 4, respectively).

${ }^{1} \mathrm{H}$ NMR (300 MHz, $\left.\mathrm{CDCl}_{3}\right) \delta 8.14(\mathrm{~s}, 1 \mathrm{H}), 8.12(\mathrm{~s}, 1 \mathrm{H}), 7.85(\mathrm{~s}, 1 \mathrm{H}), 7.81(\mathrm{~s}, 1 \mathrm{H}), 3.41(\mathrm{~s}, 2 \mathrm{H}), 2.49$ $(\mathrm{s}, 12 \mathrm{H}), 1.50(\mathrm{~s}, 6 \mathrm{H})$.

${ }^{13} \mathrm{C}$ NMR $\left(75 \mathrm{MHz}, \mathrm{CDCl}_{3}\right) \delta 160.1,153.0,145.7,144.4,141.9$ (2C), 141.8, 141.7, 141.6, 141.3, $140.4,140.3,129.8,129.6,128.2,127.7,46.0,27.7,20.8,20.6$ (3C).

HRMS (ESI+) calcd for $\mathrm{C}_{24} \mathrm{H}_{25} \mathrm{~N}_{4}[\mathrm{M}+\mathrm{H}]^{+}$369.2079. Found 369.2056. 


\section{2-(Phenazin-2-yloxy)ethanol (5)}<smiles>OCCOc1ccc2nc3ccccc3nc2c1</smiles>

Pale yellow solid (156 mg, 65\% when TFA was used as a catalyst; $163 \mathrm{mg}, 68 \%$ in the absence of TFA; Scheme 3).

${ }^{1} \mathrm{H}$ NMR (300 MHz, $\left.\mathrm{CDCl}_{3}\right) \delta 8.17-8.09(\mathrm{~m}, 2 \mathrm{H}), 8.01$ (d, $\left.J=9.4 \mathrm{~Hz}, 1 \mathrm{H}\right), 7.78-7.68(\mathrm{~m}, 2 \mathrm{H}), 7.39-$ $7.33(\mathrm{~m}, 2 \mathrm{H}), 4.27(\mathrm{t}, J=4.5 \mathrm{~Hz}, 2 \mathrm{H}), 4.08(\mathrm{t}, J=4.5 \mathrm{~Hz}, 2 \mathrm{H}), 3.29$ (broad s, $1 \mathrm{H})$.

${ }^{13} \mathrm{C}$ NMR $\left(75 \mathrm{MHz}, \mathrm{CDCl}_{3}\right) \delta 160.4,145.0,143.4,142.1,140.8,130.9,130.8,129.7,129.4,128.9$, 126.5, 105.3, 70.2, 61.1 .

HRMS (ESI + ) calcd for $\mathrm{C}_{14} \mathrm{H}_{13} \mathrm{~N}_{2} \mathrm{O}_{2}[\mathrm{M}+\mathrm{H}]^{+}$241.0977. Found 241.0948.

\section{Pyrido[2,3-a]phenazine (6)}<smiles>c1cnc2c(c1)ccc1nc3ccccc3nc12</smiles>

Pale yellow solid (106 mg, 46\%, Scheme 4).

${ }^{1} \mathrm{H}$ NMR (500 MHz, $\mathrm{CDCl}_{3}$ ) $\delta 8.179 .05$ (dd, $\left.J=4.4,1.6 \mathrm{~Hz}, 1 \mathrm{H}\right), 8.49-8.46$ (m, 1H), 8.12-8.09 (m, $1 \mathrm{H}), 7.99(\mathrm{dd}, J=8.0,1.6 \mathrm{~Hz}, 1 \mathrm{H}), 7.84(\mathrm{~d}, J=9.3 \mathrm{~Hz}, 1 \mathrm{H}), 7.76-7.70(\mathrm{~m}, 3 \mathrm{H}), 7.47(\mathrm{dd}, J=8.9,4.4$ $\mathrm{Hz}, 1 \mathrm{H})$.

${ }^{13} \mathrm{C}$ NMR $\left(75 \mathrm{MHz}, \mathrm{CDCl}_{3}\right) \delta 150.4,146.3,144.6,143.2,142.5,141.9,136.0,130.9$ (2C), 130.6, $130.4,129.1,128.6,128.5,124.0$.

HRMS (ESI+) calcd for $\mathrm{C}_{15} \mathrm{H}_{10} \mathrm{~N}_{3} \mathrm{O}[\mathrm{M}+\mathrm{H}]^{+}$232.0875. Found 232.0859.

\section{Pyrido[4,3-a]phenazine (7)}<smiles></smiles>

Pale yellow solid (95 mg, 41\%, Scheme 4).

${ }^{1} \mathrm{H}$ NMR $\left(300 \mathrm{MHz}, \mathrm{CDCl}_{3}\right) \delta 9.25(\mathrm{~s}, 1 \mathrm{H}), 9.06(\mathrm{~d}, J=5.5 \mathrm{~Hz}, 1 \mathrm{H}), 8.92(\mathrm{~d}, J=5.5 \mathrm{~Hz}, 1 \mathrm{H}), 8.36-$ $8.30(\mathrm{~m}, 1 \mathrm{H}), 8.29-8.23(\mathrm{~m}, 1 \mathrm{H}), 8.02(\mathrm{~s}, 2 \mathrm{H}), 7.93-7.85(\mathrm{~m}, 2 \mathrm{H})$.

${ }^{13} \mathrm{C}$ NMR $\left(75 \mathrm{MHz}, \mathrm{CDCl}_{3}\right) \delta 150.8,147.5,144.5,143.9,142.3,141.0,136.5,131.4,130.7,130.4$, 130.1, 129.6, 129.1, 127.9, 118.2.

HRMS (ESI+) calcd for $\mathrm{C}_{15} \mathrm{H}_{10} \mathrm{~N}_{3} \mathrm{O}[\mathrm{M}+\mathrm{H}]^{+}$232.0875. Found 232.0869. 


\title{
Crystallographic data collection, structure determination and refinement
}

X-ray structure determination of 3aa was performed using a Rigaku diffractometer constituted by a MM007 HF rotating-anode generator, delivering copper radiation through Osmic CMF confocal optics, and a Rapid II curved Image Plate detector, on a colourless crystalline stick. Data collection was carried out with the CrystalClear 2.0 package implementing Fs_process software for the data reduction. ${ }^{[1]}$ The structure was solved by intrinsic phasing methods ${ }^{[2]}$ and its refinement was carried out by full-matrix least-squares methods ${ }^{[3]}$ on 227 parameters, using the following weighted scheme: $w=1 /\left[\sigma^{2}\left(\mathrm{~F}_{\mathrm{o}}^{2}\right)+\right.$ $\left.(0.1718 \mathrm{P})^{2}\right]$ with $\mathrm{P}=\left[\max \left(\mathrm{F}_{\mathrm{o}}^{2}, 0\right)+2 \mathrm{~F}_{\mathrm{o}}^{2}\right] / 3$. The dataset was cut off at the $0.86 \AA$ resolution limit since half of the data were below the $2 *$ sigma(I) level (that crystal was a weak diffractor). $\mathrm{H}$ atoms were refined using a riding model, with $\mathrm{C}-\mathrm{H}=0.95$ (aromatic) and 0.99 $\AA\left(\mathrm{CH}_{2}\right)$, and with $U_{\text {iso }}(\mathrm{H})=1.2 U_{\text {eq }}(\mathrm{C})$. Non-hydrogen atoms improved by anisotropic refinement. Crystal data, data collection and structure refinement details are summarized in Table 1. One quasi- planar molecule of 3aa (rms deviation for the 22 atoms is $0.055 \AA$ ) was found in the asymmetric unit of the non-centrosymmetric space group P $2_{1}$, together with a solvent dichloromethane molecule pointing both hydrogens into the concave curvature of the molecule toward N3 in general position (distance of $2.71 \AA$ ) and N1 of a x-translated $(+1)$

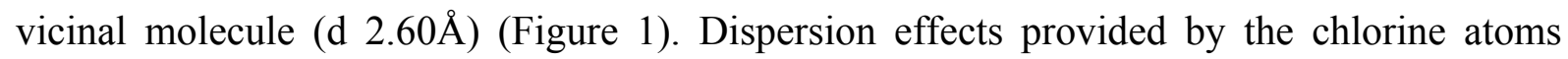
helped us to determine the absolute structure ${ }^{[4]}$ via a BASF/TWIN refinement of the Flack parameter $^{[5]}(0.11(8))$, i.e. to specify the orientation of the achiral quinoxalinophenazine molecule, featuring a croissant shape, under the operation of inversion, which is equivalent here to a pure rotation.

CCDC 1879520 (compound 3aa) contains the supplementary crystallographic data for this paper. These data can be obtained free of charge from The Cambridge Crystallographic Data Centre via www.ccdc.cam.ac.uk/data_request/cif.

\author{
References \\ Rigaku. (2009) CrystalClear-SM Expert 2.0 r4 Rigaku Corporation, Tokyo, Japan. \\ Sheldrick, G. M. (2015). Acta Crystallogr., A71, 3-8. \\ Sheldrick, G. M. (2015). Acta Crystallogr., C71, 3-8. \\ http://reference.iucr.org/dictionary/Absolute_structure. \\ Flack, H. D. (1983). Acta Crystallogr., A39, 876-881.
}




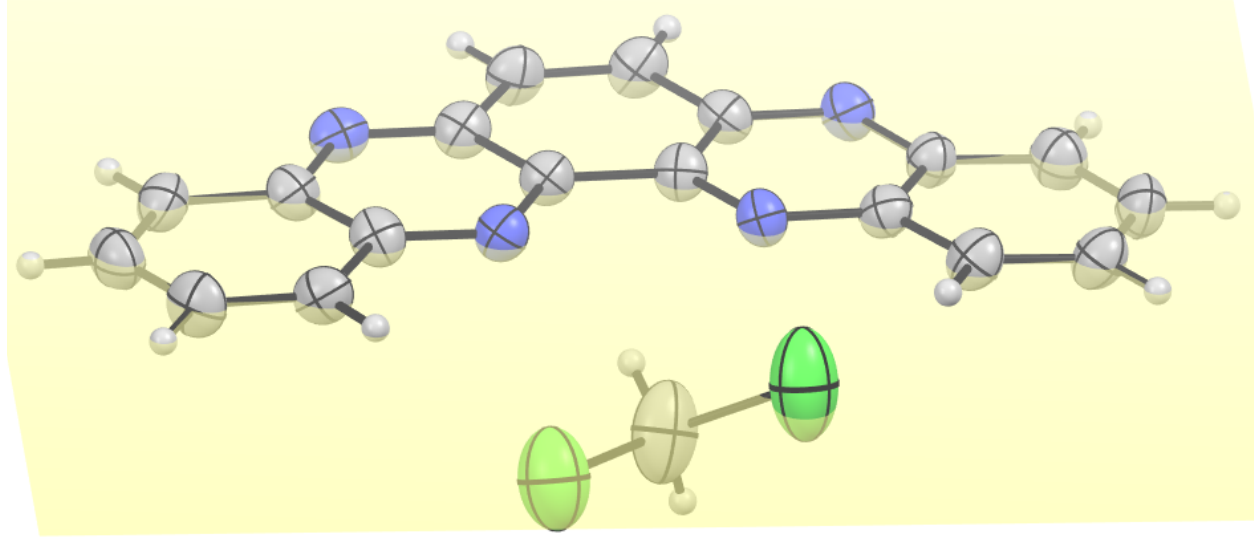

Figure 1 Ortep plot of 3aa. Ellipsoids are drawn at 30\% of probability 
Table 1. Crystal data and structure refinement for 3aa.

\begin{tabular}{|c|c|c|}
\hline Identification code & \multicolumn{2}{|l|}{$3 \mathbf{a a}$} \\
\hline Empirical formula & \multicolumn{2}{|l|}{$\mathrm{C}_{18} \mathrm{H}_{10} \mathrm{~N}_{4}, \mathrm{CH}_{2} \mathrm{Cl}_{2}$} \\
\hline Formula weight & \multicolumn{2}{|l|}{367.23} \\
\hline Temperature & \multicolumn{2}{|l|}{ 293(2) K } \\
\hline Wavelength & \multicolumn{2}{|l|}{$1.54187 \AA$} \\
\hline Crystal system & \multicolumn{2}{|l|}{ Monoclinic } \\
\hline Space group & \multicolumn{2}{|l|}{$\mathrm{P} 22_{1}$} \\
\hline \multirow[t]{3}{*}{ Unit cell dimensions } & $\mathrm{a}=3.8134(3) \AA$ & $\alpha=90^{\circ}$ \\
\hline & $\mathrm{b}=19.2011(11) \AA$ & $\beta=92.869(6)^{\circ}$. \\
\hline & $\mathrm{c}=11.0869(8) \AA$ & $\gamma=90^{\circ}$ \\
\hline Volume & \multicolumn{2}{|l|}{$810.78(10) \AA^{3}$} \\
\hline $\mathrm{Z}$ & \multicolumn{2}{|l|}{2} \\
\hline Density (calculated) & \multicolumn{2}{|l|}{$1.504 \mathrm{Mg} / \mathrm{m}^{3}$} \\
\hline Absorption coefficient & \multicolumn{2}{|l|}{$3.672 \mathrm{~mm}^{-1}$} \\
\hline $\mathrm{F}(000)$ & \multicolumn{2}{|l|}{376} \\
\hline Crystal size & \multicolumn{2}{|c|}{$0.34 \times 0.12 \times 0.10 \mathrm{~mm}^{3}$} \\
\hline () range for data collection & \multicolumn{2}{|l|}{3.992 to $63.692^{\circ}$} \\
\hline Index ranges & \multicolumn{2}{|c|}{$-4 \leq \mathrm{h} \leq 4,-22 \leq \mathrm{k} \leq 20,-12 \leq 1 \leq 12$} \\
\hline Reflections collected & \multicolumn{2}{|c|}{6889} \\
\hline Independent reflections & \multicolumn{2}{|c|}{$2443[\mathrm{R}(\mathrm{int})=0.0392]$} \\
\hline Completeness to $\theta=67.687^{\circ}$ & \multicolumn{2}{|l|}{$99.9 \%$} \\
\hline Absorption correction & \multicolumn{2}{|c|}{ Semi-empirical from equivalents } \\
\hline Max. and min. transmission & \multicolumn{2}{|c|}{1.000 and 0.756} \\
\hline Refinement method & \multicolumn{2}{|c|}{ Full-matrix least-squares on $F^{2}$} \\
\hline Data / restraints / parameters & \multicolumn{2}{|l|}{$2442 / 1 / 227$} \\
\hline Goodness-of-fit on $\mathrm{F}^{2}$ & \multicolumn{2}{|l|}{0.988} \\
\hline Final $\mathrm{R}$ indices $[I>2 \sigma(I)]$ & \multicolumn{2}{|c|}{$\mathrm{R} 1=0.0811, \mathrm{wR} 2=0.2081$} \\
\hline $\mathrm{R}$ indices (all data) & \multicolumn{2}{|c|}{$\mathrm{R} 1=0.1464, \mathrm{wR} 2=0.276$} \\
\hline Absolute structure & \multicolumn{2}{|l|}{$0.11(8)$} \\
\hline Largest diff. peak and hole & \multicolumn{2}{|l|}{0.36 and -0.48 e. $\AA^{-3}$} \\
\hline CCDC deposit number & \multicolumn{2}{|l|}{1879520} \\
\hline
\end{tabular}




\section{Crystallographic data collection, structure determination and refinement}

X-ray diffraction data for pyrido[2,3-a]phenazine (6) were measured at room temperature using a RIGAKU XtaLabPro diffractometer equipped with a Mo microfocus sealed tube generator coupled to a double-bounce confocal Max-Flux ${ }^{\circledR}$ multilayer optic and a HPAD PILATUS3R 200K detector. CrysAlisPro 1.171.39.46 ${ }^{[1]}$ was employed for the data processing, with SCALE3 ABSPACK scaling algorithm implemented for the empirical absorption correction using spherical harmonics. Regarding the thin needles obtained for pyrido[4,3-a]phenazine (7), the data collection was carried out at $193 \mathrm{~K}$ using a RIGAKU diffractometer constituted by a MM007 HF rotating-anode generator, delivering copper radiation through Osmic CMF confocal optics, and a Rapid II curved Image Plate detector. Fs_process ${ }^{[2]}$ software under the CrystalClear $2.0^{[3]}$ suite was used to integrate and scale the data, applying multi-scan $R E Q A B{ }^{[2]}$ for the absorption correction. The reduced data set was cut off at the $0.86 \AA$ resolution limit since little diffraction signal could be detected beyond the $i U C R$ claimed resolution limit despite intense radiation and long exposure. Both structures were solved by intrinsic phasing methods (SHELXT program), ${ }^{[4]}$ then refined by full-matrix least-squares methods on $F^{2}$ using SHELX-L. ${ }^{[5]}$ All non-hydrogen atoms of the molecules of interest improved by anisotropic refinement. Most of the $\mathrm{H}$ atoms were identified in Fourier difference maps. Aromatic $\mathrm{H}$ atoms bound to carbon atoms were positioned geometrically and refined with $U_{\text {iso }}$ set to $1.2 U_{\mathrm{eq}}(\mathrm{C})$ of the parent carbon atom. Whereas there is only one molecule of pyrido[4,3-a]phenazine (7) in the asu of the monoclinic form, two independent molecules of pyrido[2,3-a]phenazine (6) occupy the asymmetric unit cell of the non-centrosymmetric orthorhombic crystal, making a relative dihedral angle of $73.5^{\circ}$ and two h-bonds including a bifurcated one involving $\mathrm{C} 3$ and $\mathrm{C} 9$ at the position $\mathrm{x}, \mathrm{y}+1, \mathrm{z}($ resp. $\mathrm{x}, \mathrm{y}, \mathrm{z}$ ) and the two $\mathrm{N}$ atoms placed inside the concavity of the molecule at $\mathrm{x}, \mathrm{y}, \mathrm{z}$ (resp. $0.5-\mathrm{x}, \mathrm{y}, 0.5+\mathrm{z}$ ) (distance H3 ...N2 is $2.93 \AA, \mathrm{H} 9 \ldots \mathrm{N} 1,3.07 \AA$ and $\mathrm{H} 9 \ldots \mathrm{N} 2,3.23 \AA$ ). Each molecule of the asu piles up along the $\boldsymbol{b}$ direction with a planar separation of $3.39 \AA$. In the monoclinic unit cell, the molecules related by a gliding mirror or an helicoidal two-fold axis are quasi-orthogonal to each other making $\mathrm{C} 15-\mathrm{H} 15 \ldots$

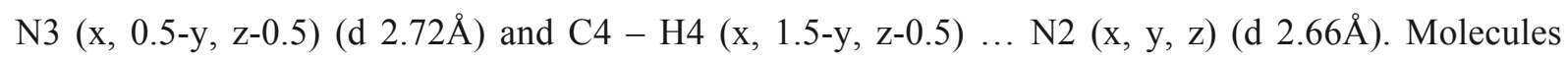
translated along the $\boldsymbol{b}$ direction are stacking with a plane separation distance of $3.41 \AA$ and paired

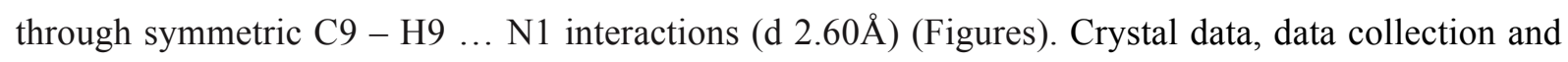
structure refinement details are summarized in Table 1.

CCDC 1918383 and 1938417 (compounds pyrido[2,3-a]phenazine (6), and pyrido[4,3-a]phenazine (7) respectively) contain the supplementary crystallographic data for this paper. These data can be obtained free of charge from The Cambridge Crystallographic Data Centre via www.ccdc.cam.ac.uk/data_request/cif.

\section{References}


1 Rigaku OD (2015). CrysAlis PRO. Rigaku Oxford Diffraction, Yarnton, Oxfordshire, England.

2 Rigaku - Higashi T. (1998) FS Process, Rigaku Corporation,Tokyo, Japan.

3 Rigaku. (2009) CrystalClear-SM Expert 2.0 r4 Rigaku Corporation, Tokyo, Japan.

4 Sheldrick, G. M. (2015). Acta Crystallogr., C71, 3-8.

5 Sheldrick, G. M. (2015). Acta Crystallogr., A71, 3-8.

6 Wolff, S. K., Grimwood, D. J., McKinnon, J. J., Jayatilaka, D. \& Spackman, M. A. (2007). Crystal Explorer. University of Western Australia, Perth, Australia. 
Table 1 Crystal data, data collection and structure refinement details for the two pyridophenazine compounds.

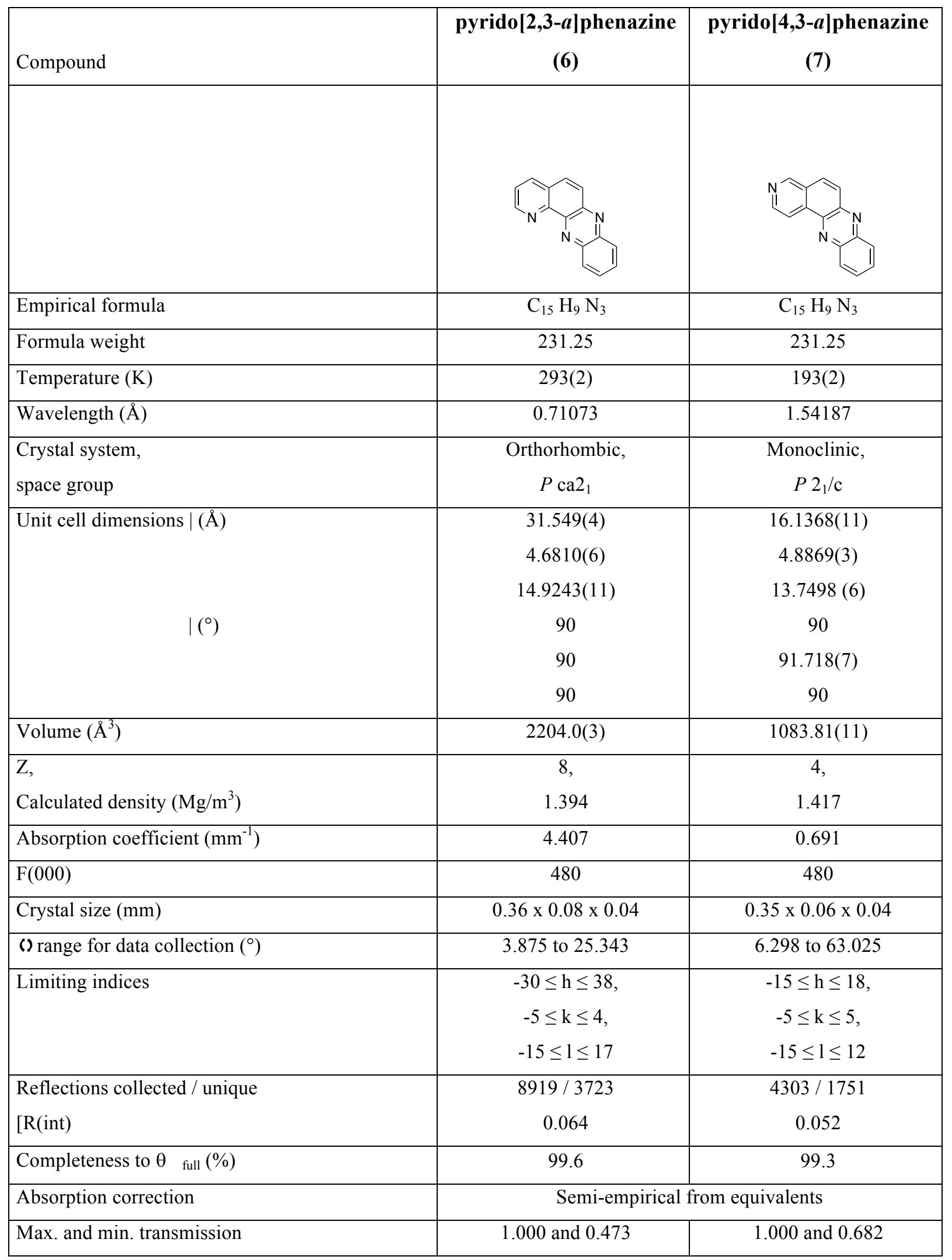




\begin{tabular}{|l|l|c|c|}
\hline \multicolumn{2}{|l|}{ Refinement method } & \multicolumn{2}{|c|}{ Full-matrix least-squares on $F^{2}$} \\
\hline \multicolumn{2}{|l|}{ Data / restraints / parameters } & $3720 / 1 / 325$ & $1743 / 0 / 163$ \\
\hline Goodness-of-fit on $F^{2}$ & R1 & 1.001 & 0.895 \\
\hline Final R indices & wR2 & 0.0467, & 0.0547, \\
$[I>2 \sigma I)]$ & R1 & 0.1024 & 0.1313 \\
\hline R indices & wR2 & 0.1140 & 0.1329, \\
$($ all data) & & 0.123 and -0.158 & 0.1868 \\
\hline Largest $\Delta$ peak and hole $\left(\mathrm{e} . \AA^{-3}\right)$ & 1918383 & 0.320 and -0.319 \\
\hline CCDC deposit number & & & 1938417 \\
\hline
\end{tabular}
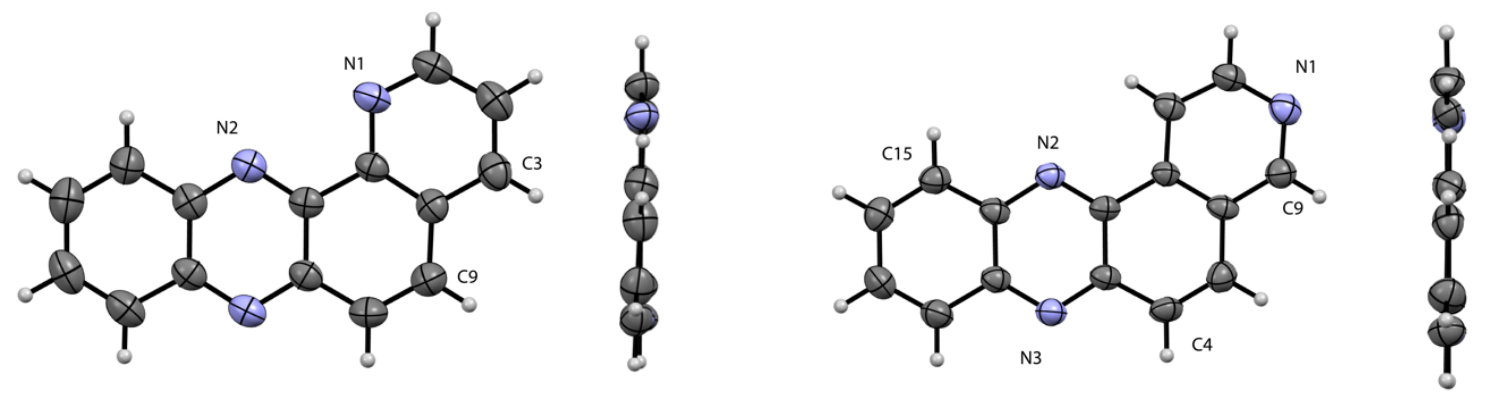

Figure 1

(left) Molecular structure of pyrido[2,3-a]phenazine (6) viewed at two $90^{\circ}$ apart (for one copy of the $a s u$ ). The rms deviation of the 18 non-H atoms in the two copies is 0.016 and $0.026 \AA$ respectively. (right) Molecular structure of pyrido[4,3-a]phenazine (7). The rms deviation of the 18 non-H atoms is $0.012 \AA$. Displacement ellipsoids are shown at the $30 \%$ probability level. $\mathrm{H}$ atoms are presented as small spheres of arbitrary radius.
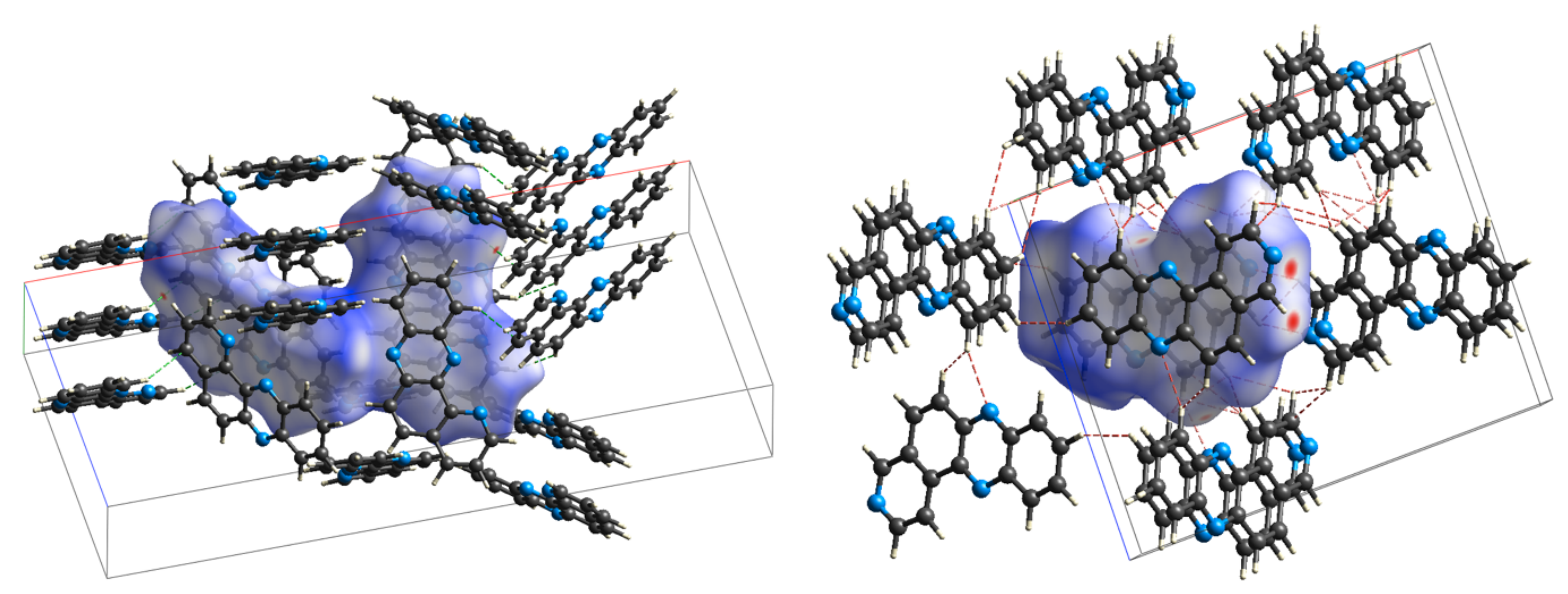

Figure 2 
View of the three-dimensional Hirshfeld surface ${ }^{6}$ mapped over $\boldsymbol{d}_{\text {norm }}$, showing the crystal packing for (left) pyrido[2,3-a]phenazine (6) and (right), pyrido[4,3-a]phenazine (7). The red spots represent contacts shorter than the sum of van der Waals radii, (white and blue regions represent intermolecular distances equal and longer to/than van der Waals contacts). The green dashed lines represent $\mathrm{C}-\mathrm{H} \ldots \pi$ contacts and the red dashed lines H... contacts shorter than $2.8 \AA$ 

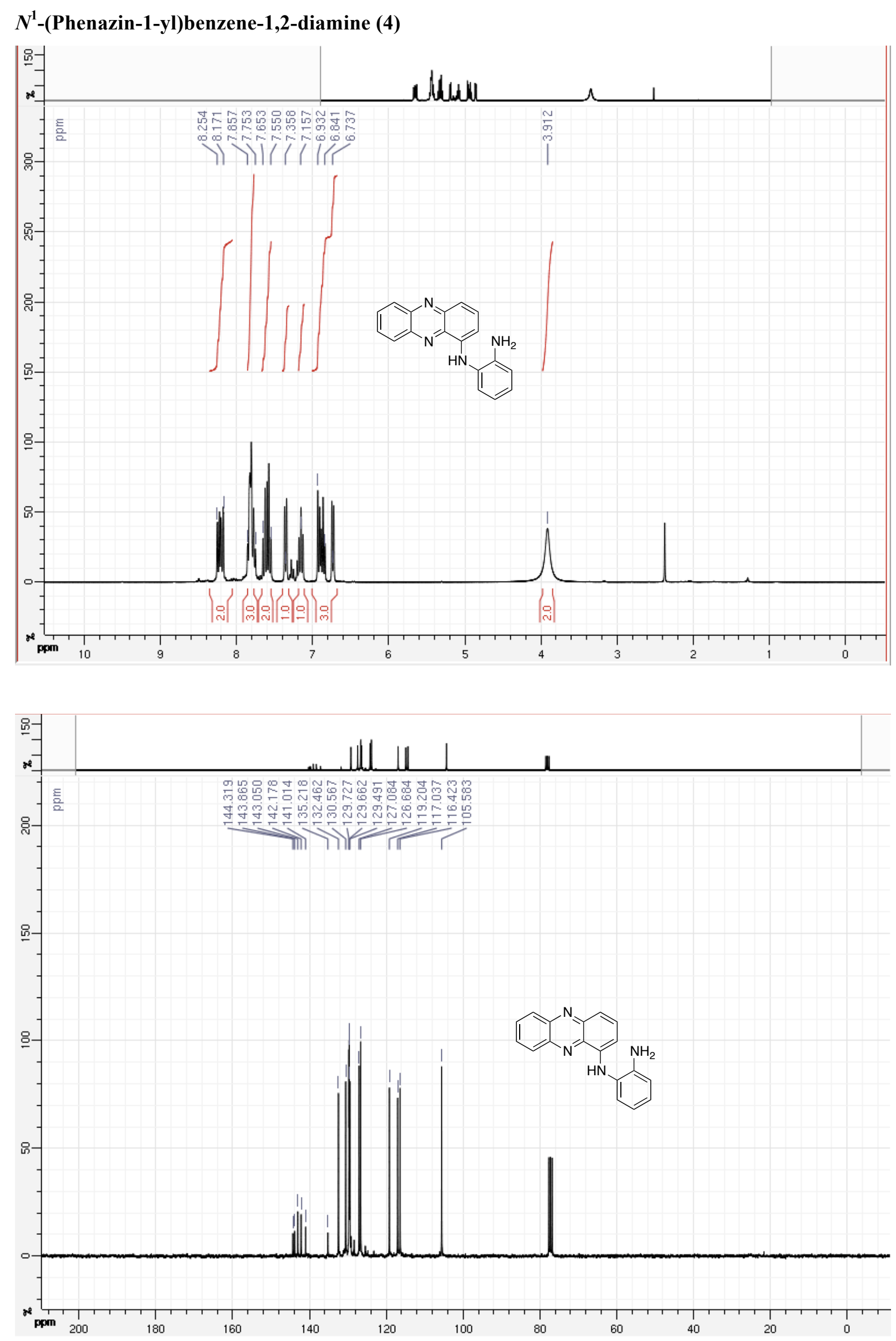

SI16 
Quinoxalino[2,3-a]phenazine (3aa)
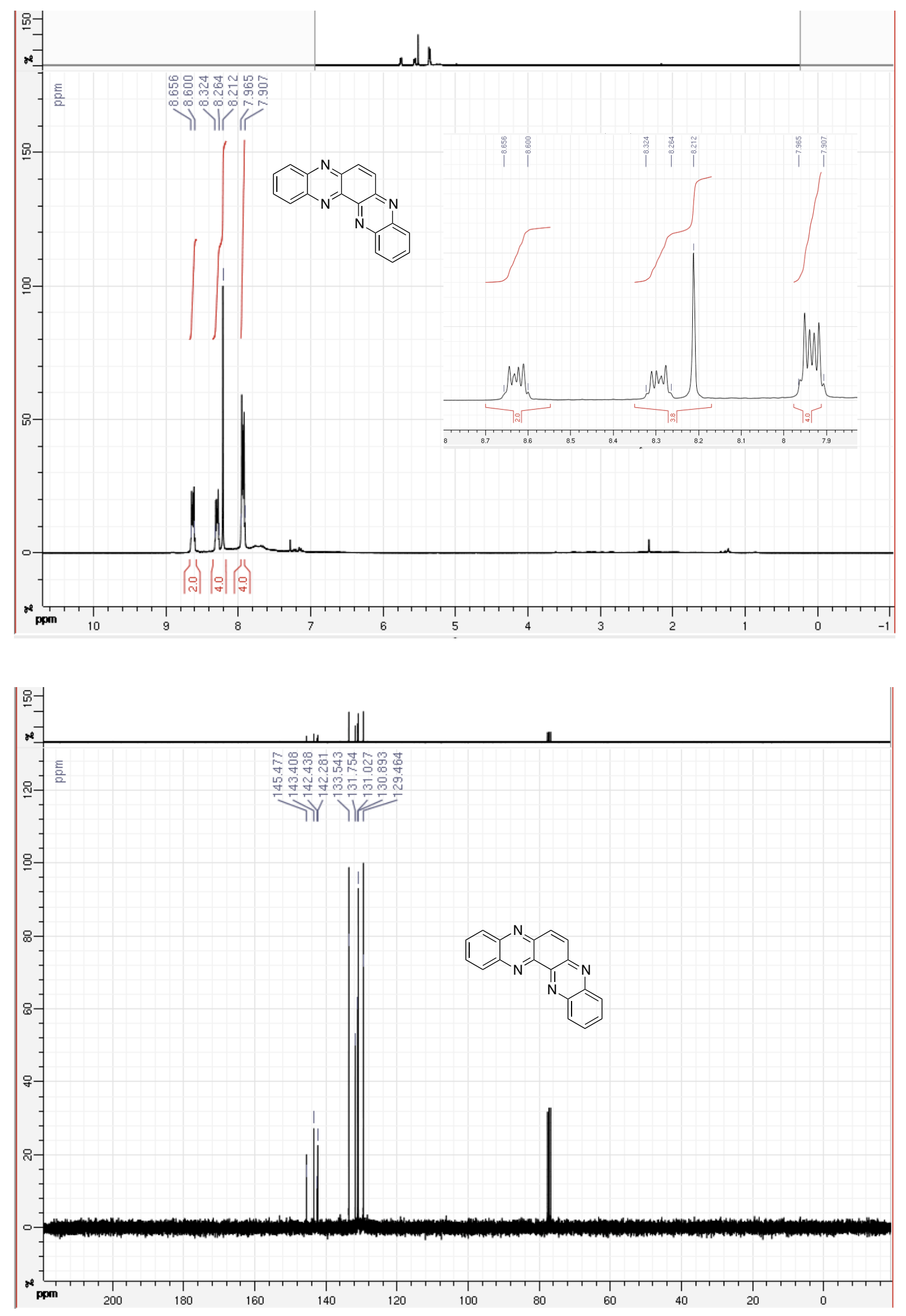

SI17 
6-Methylquinoxalino[2,3-a]phenazine (3ab)
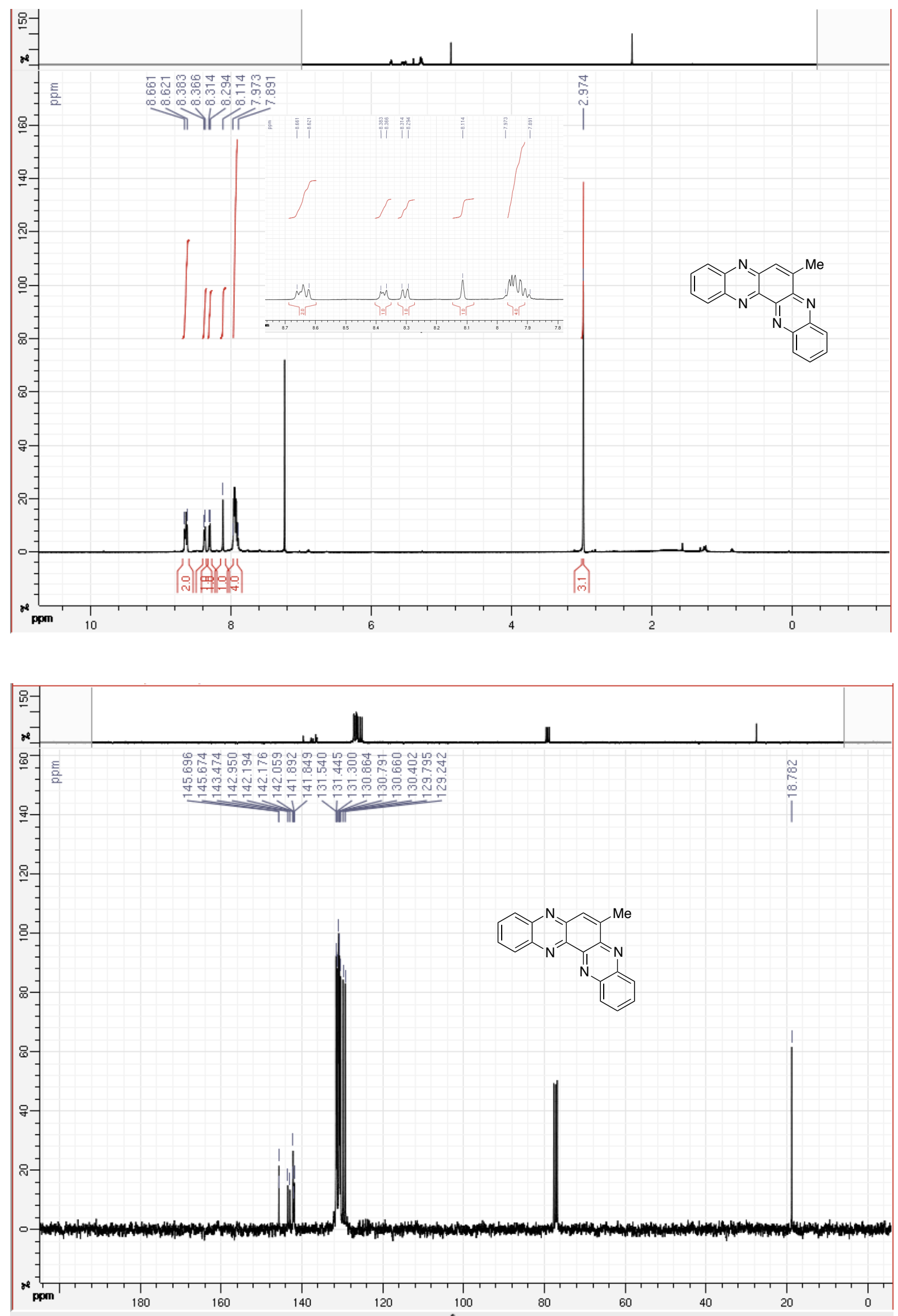
6-Propylquinoxalino $[2,3-a]$ phenazine (3ac)

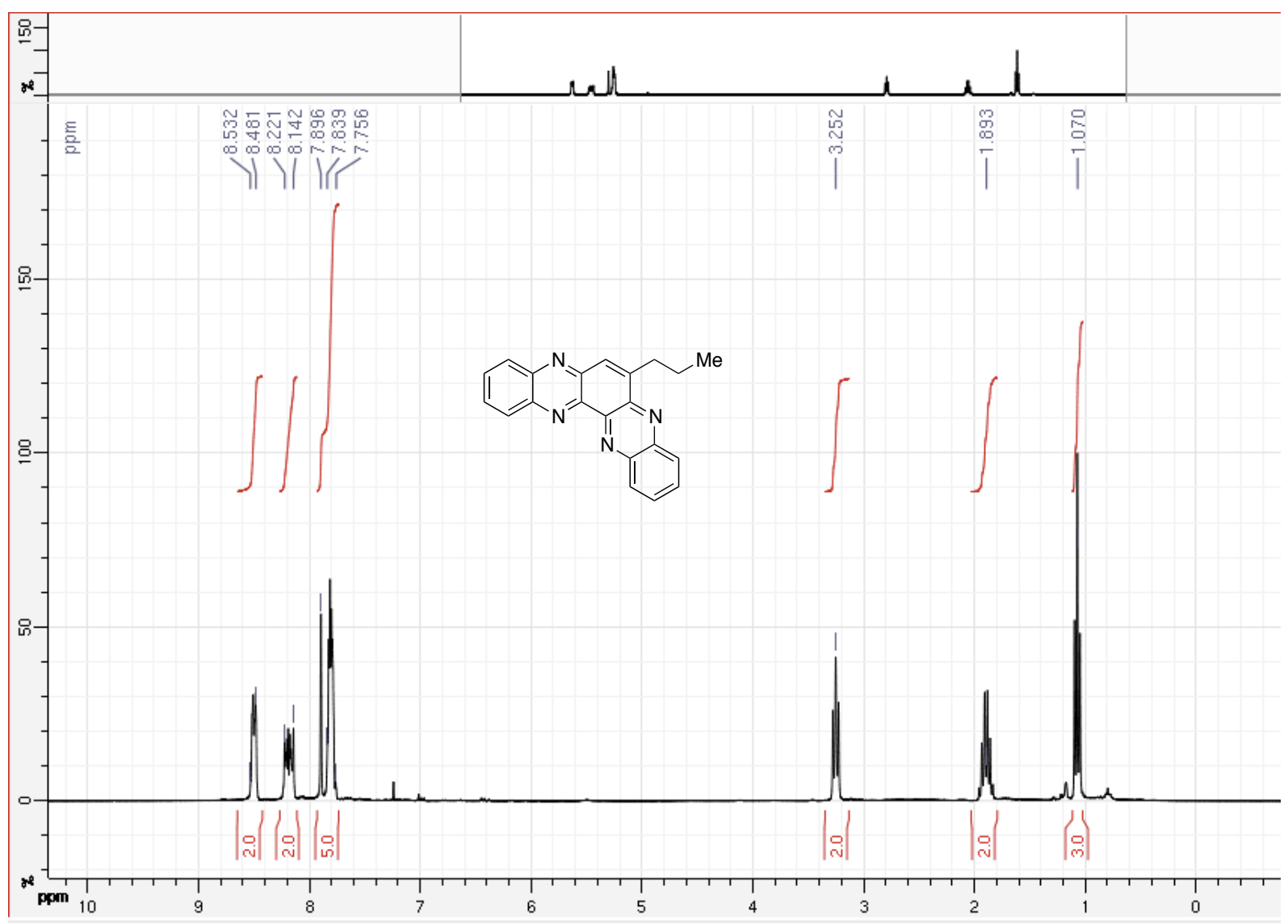

à

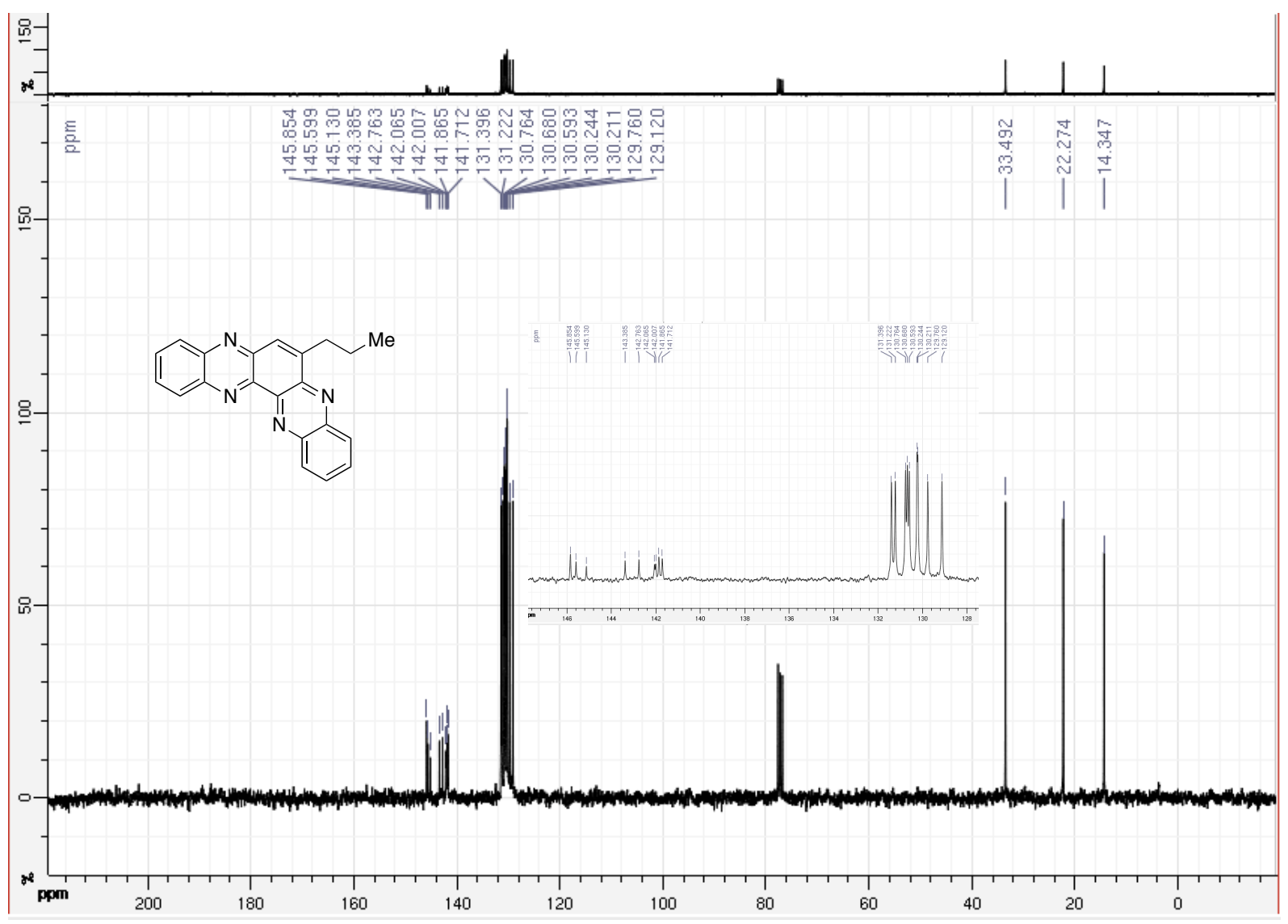

SI19 

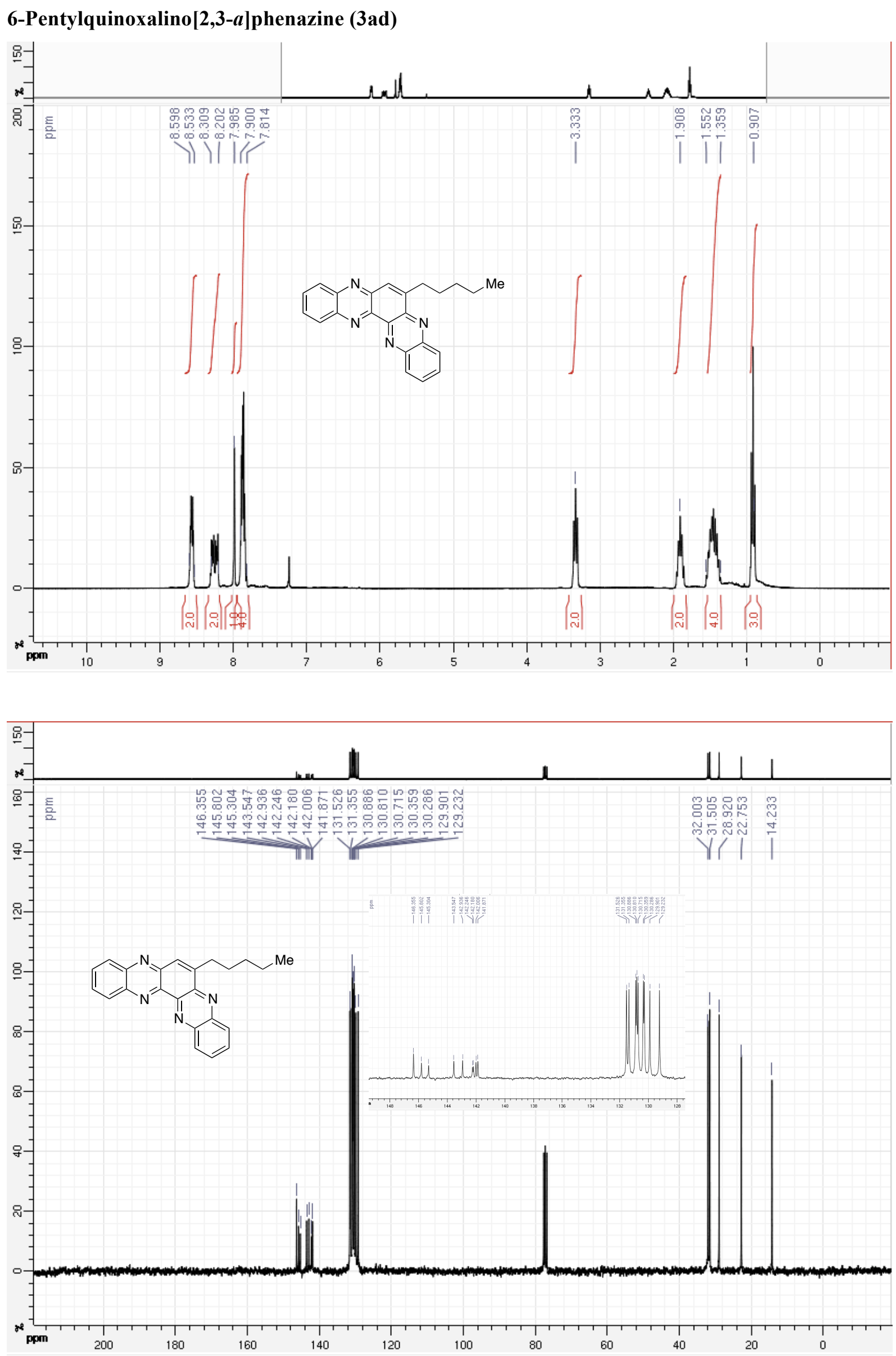

SI20 
6-Isopropylquinoxalino[2,3-a]phenazine (3ae)
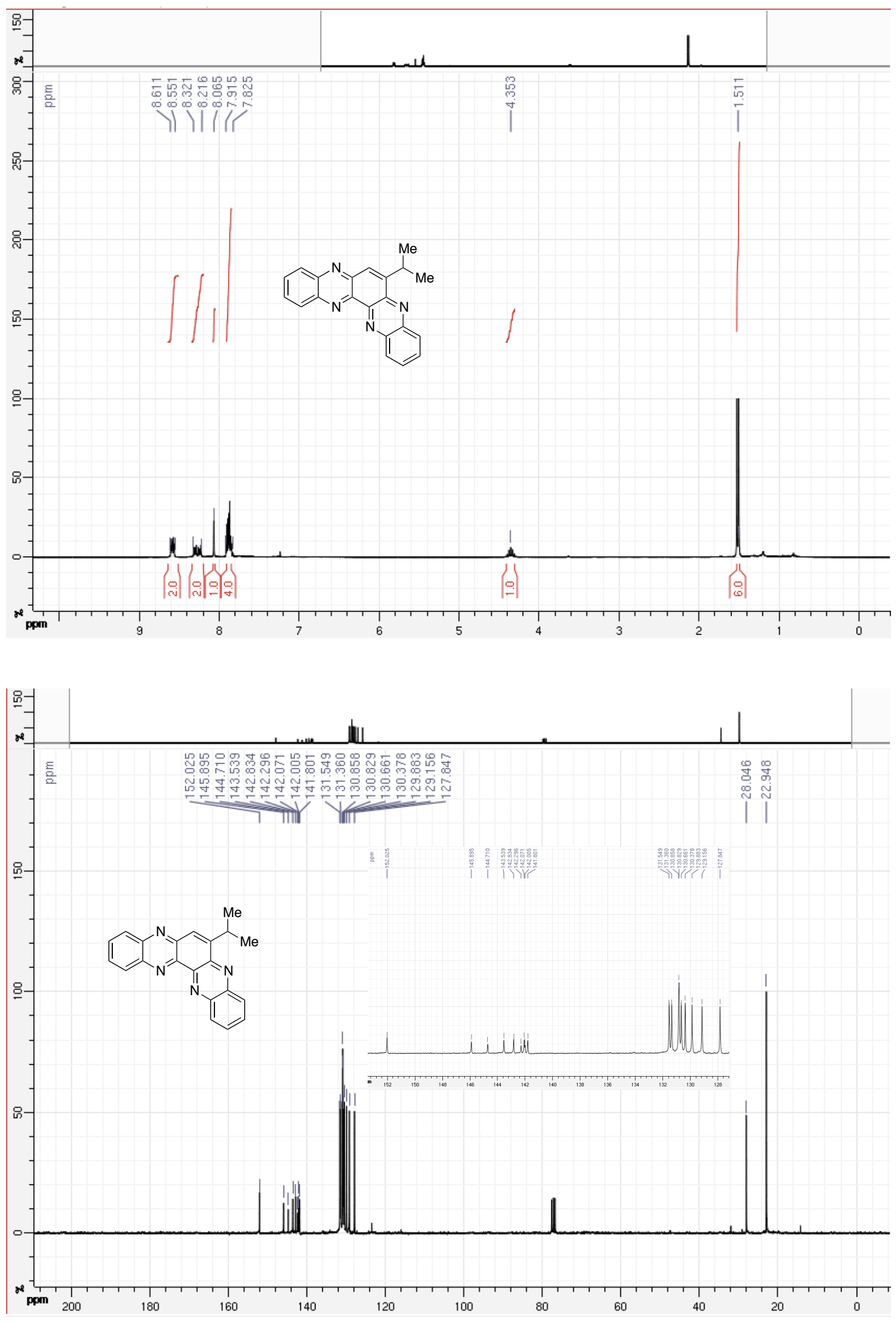

SI21 
6-(tert-Butyl)quinoxalino[2,3-a]phenazine (3af)
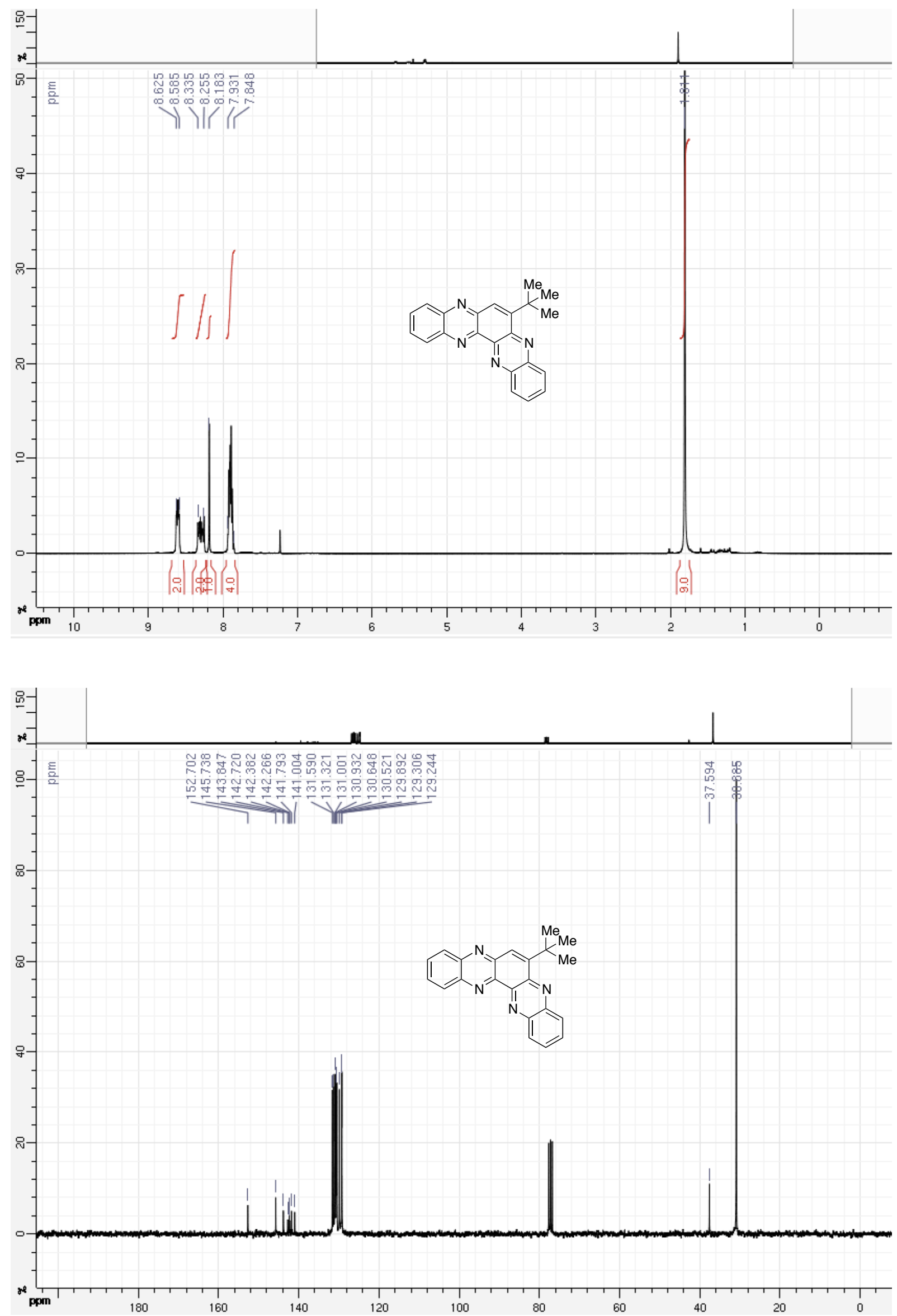
6-Phenylquinoxalino[2,3-a]phenazine (3ag)

6-Benzylquinoxalino[2,3-a]phenazine (3ah)
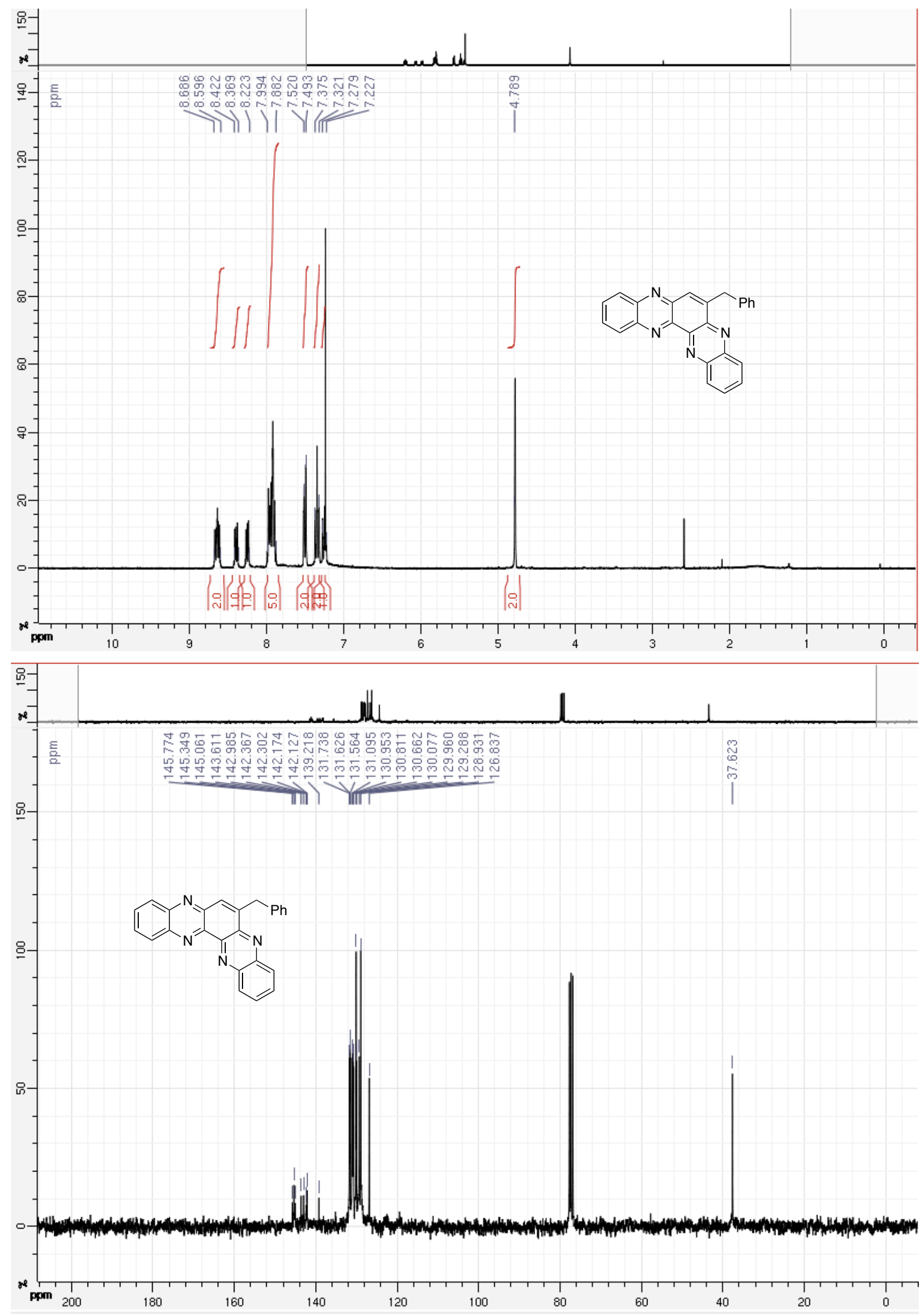
Ethyl quinoxalino[2,3-a]phenazine-6-carboxylate (3ai)
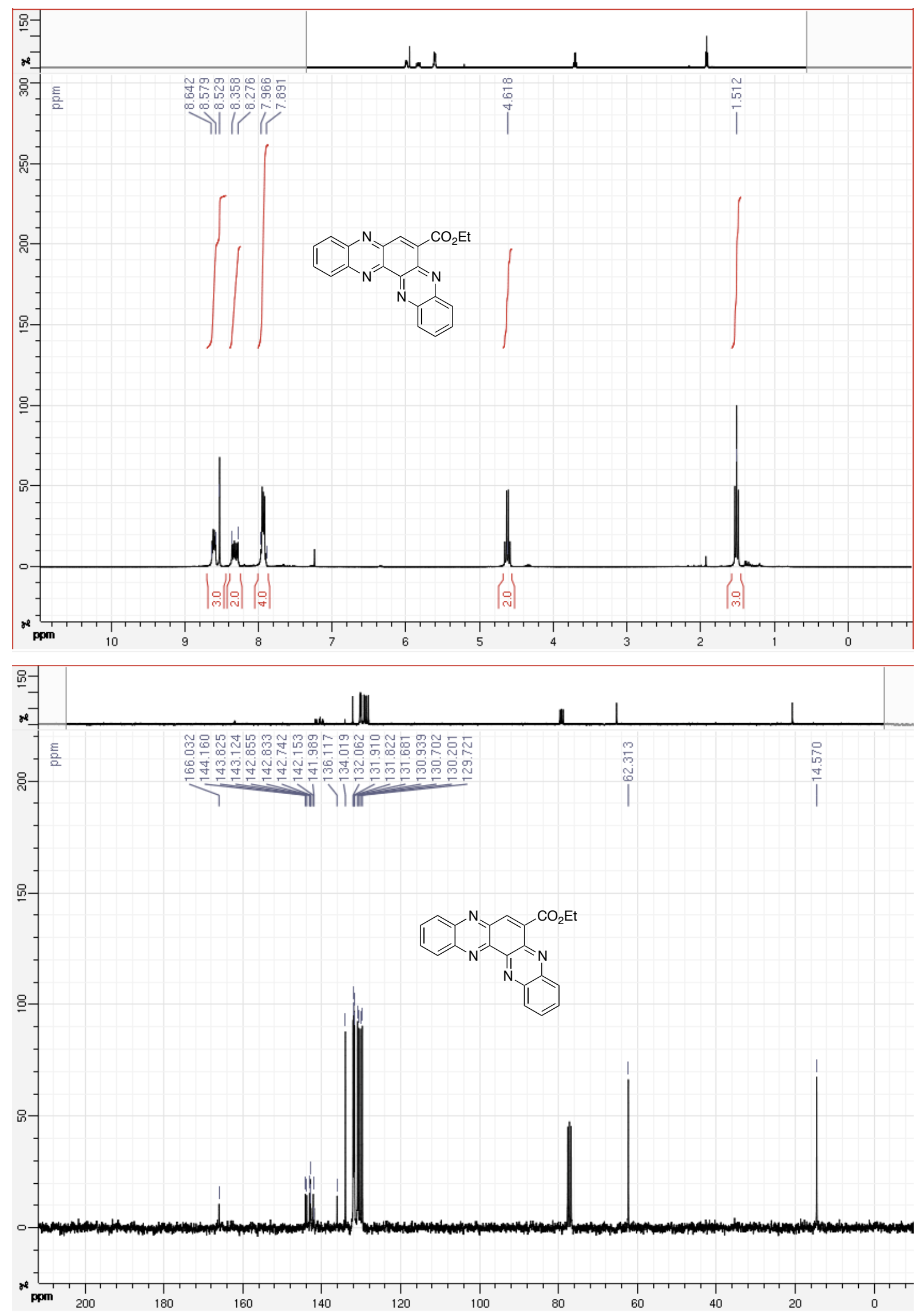
$N$-(Quinoxalino[2,3-a]phenazin-6-yl)acetamido (3aj)
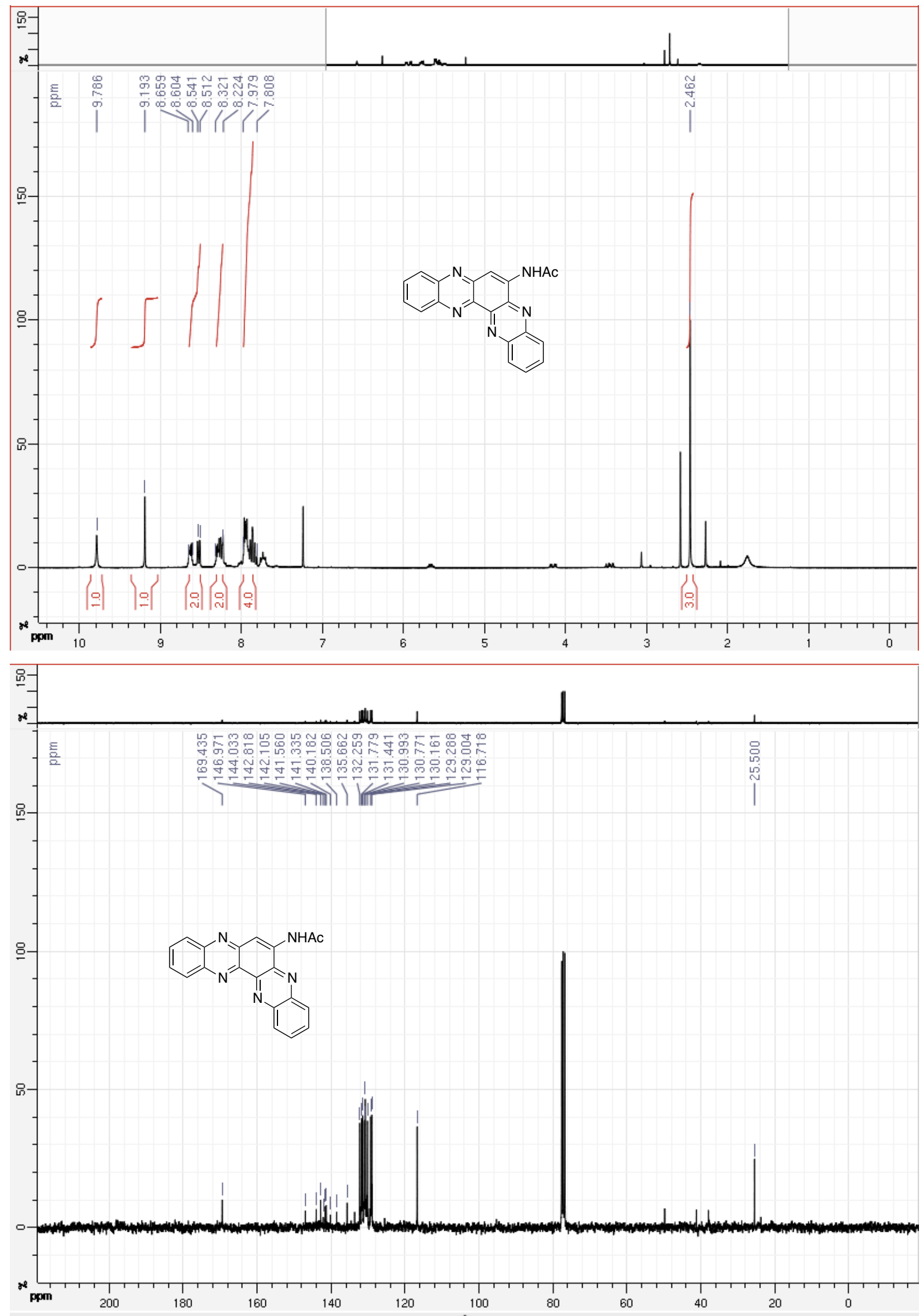
7,8-dihydro-6H-cyclohepta[1,2-b:3,4-b']diquinoxaline (3ak)
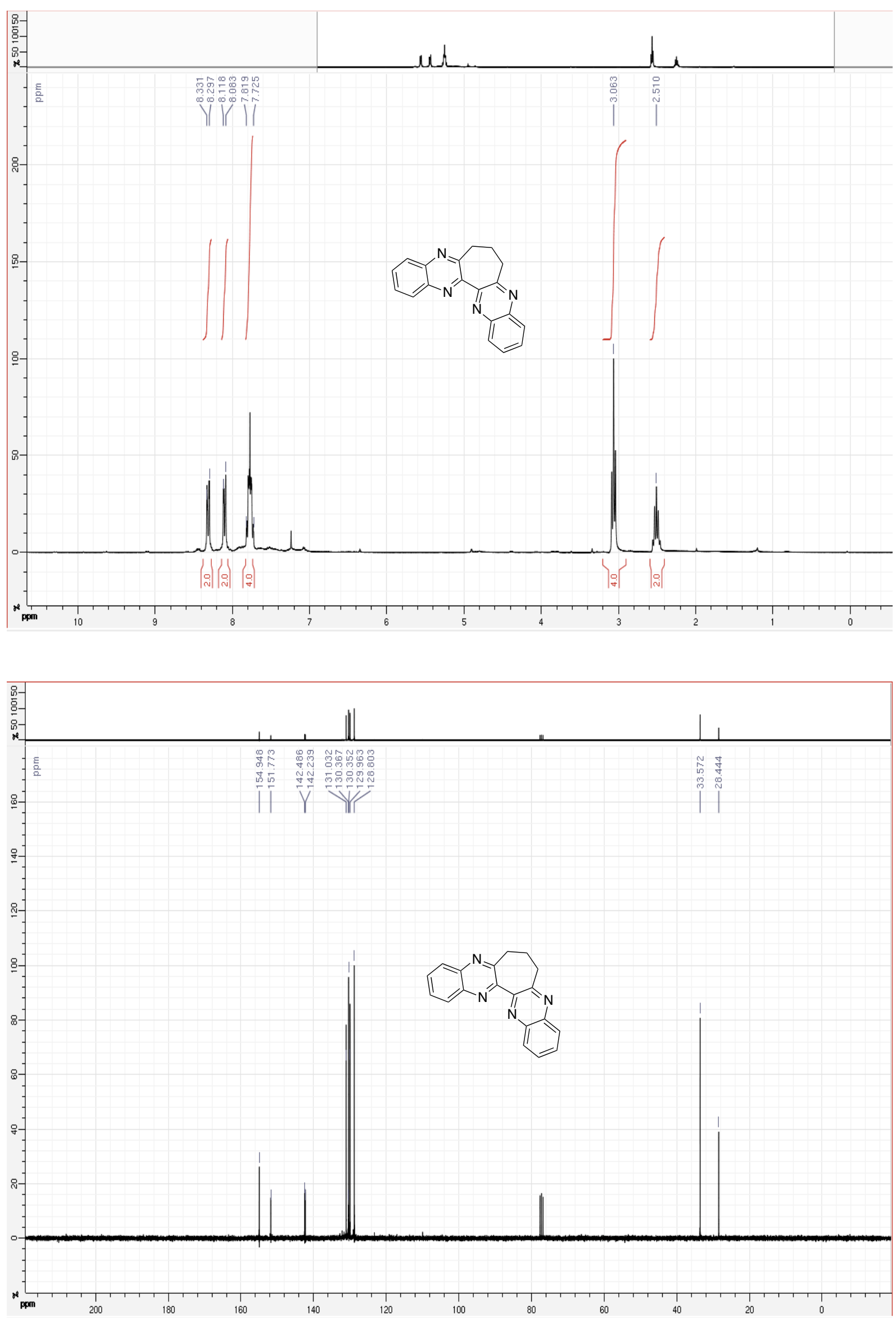

SI27 
2,3,11,12-Tetramethyl-7,8-dihydro-6H-cyclohepta[1,2-b:3,4-b']diquinoxaline (3bk)
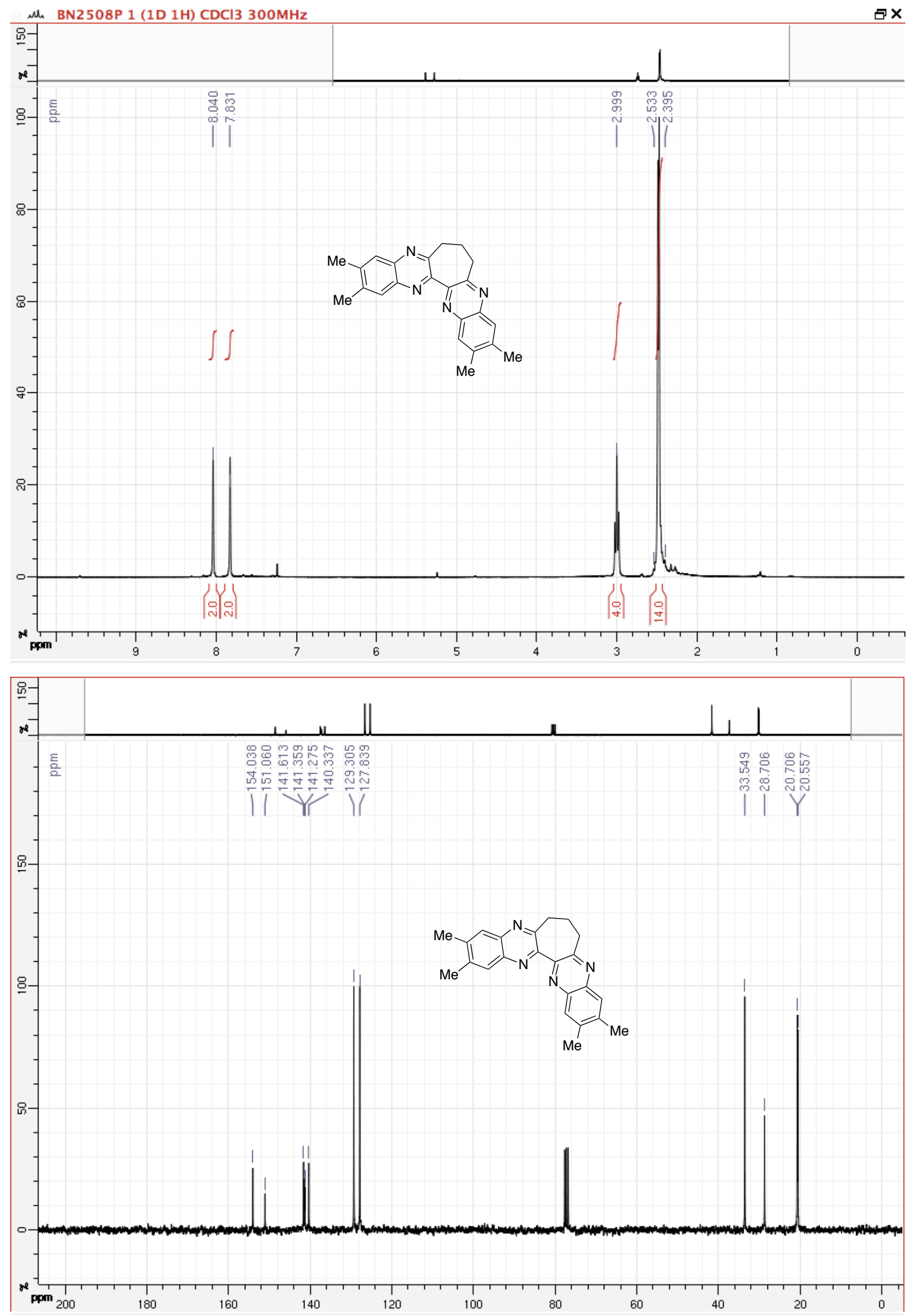
6,6-Dimethyl-6,7-dihydroquinoxalino[2,3-a]phenazine (3al)
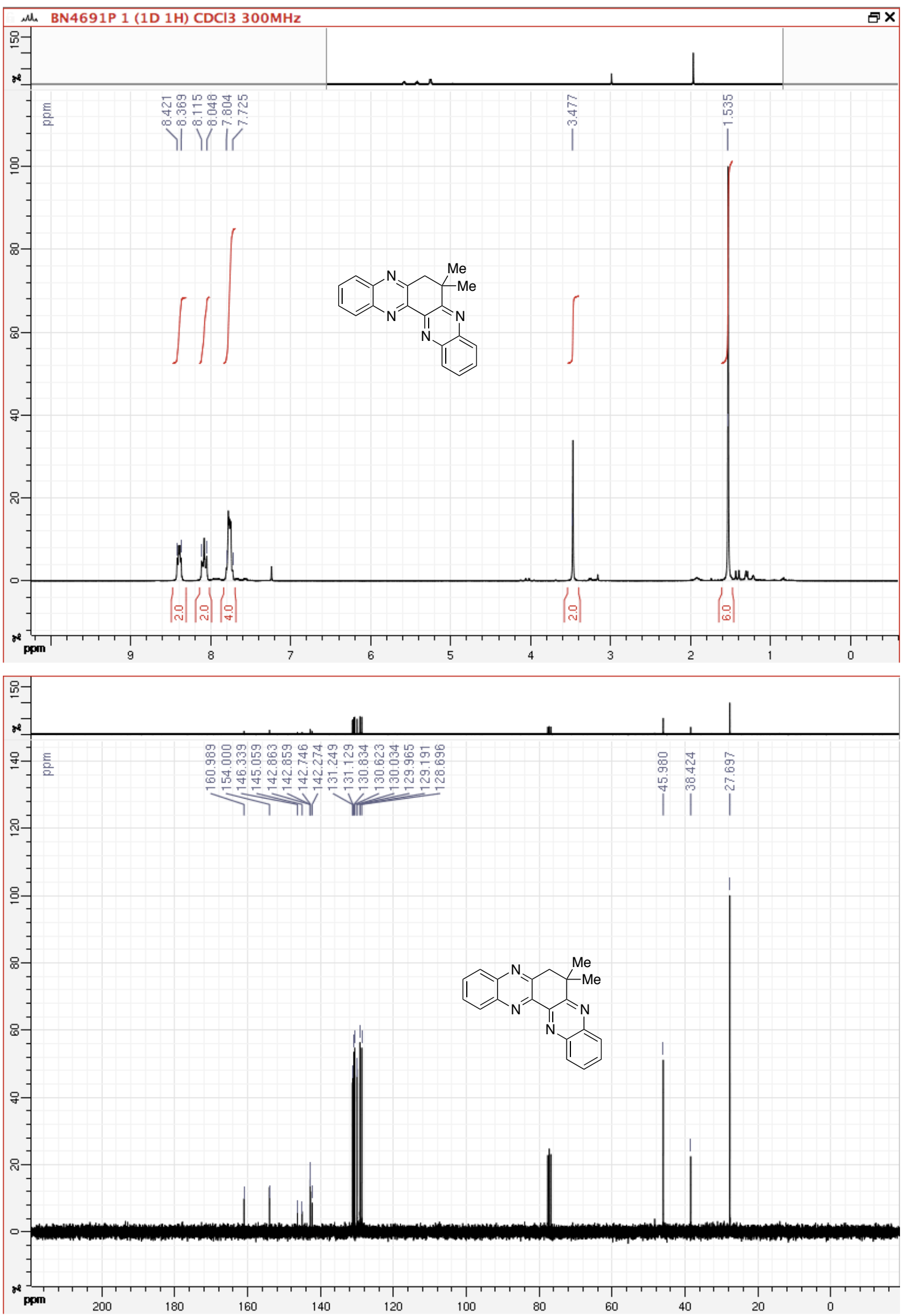
2,3,6,6,10,11-Hexamethyl-6,7-dihydroquinoxalino[2,3-a]phenazine (3bl)
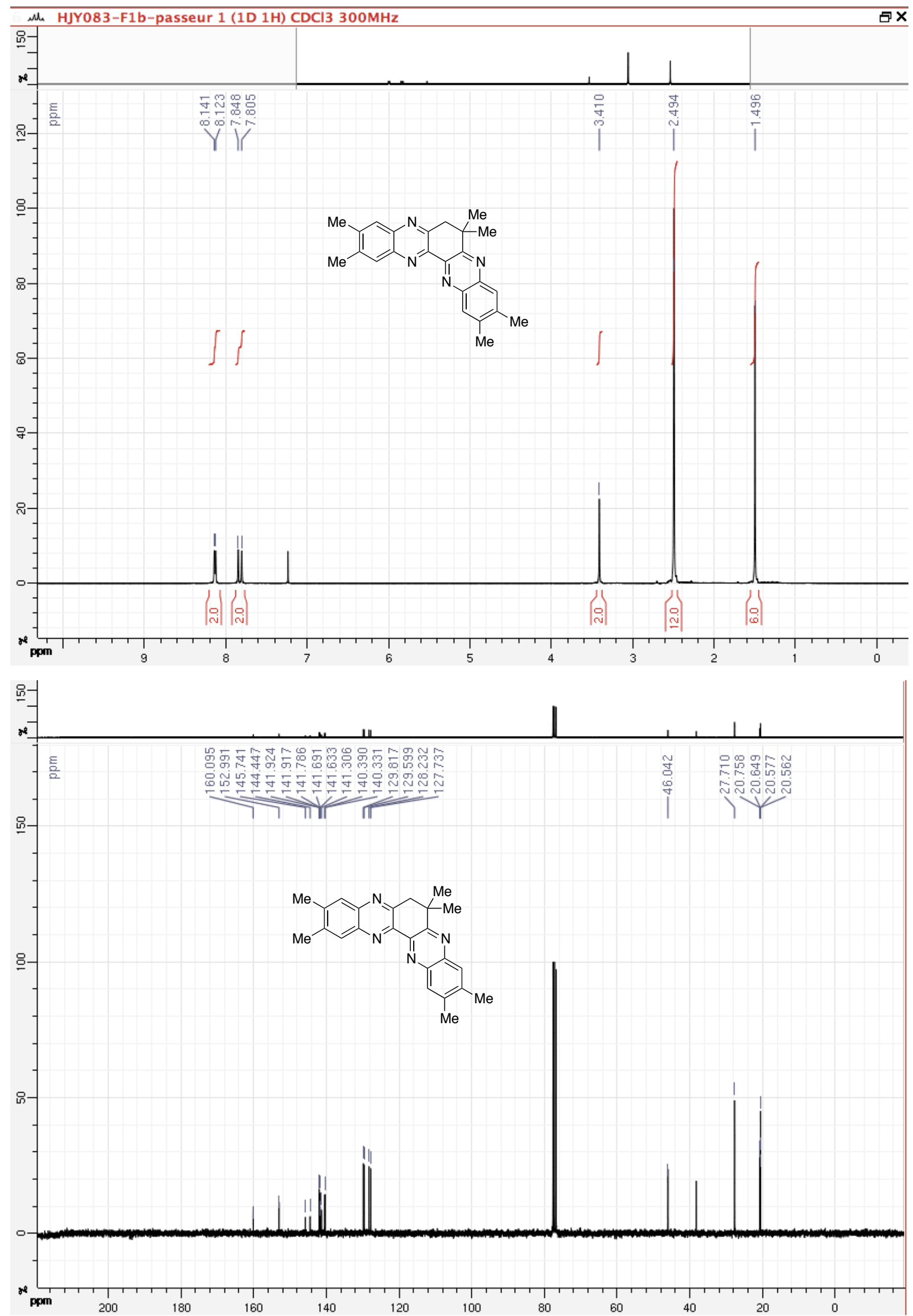
2-(Phenazin-2-yloxy)ethanol (5)
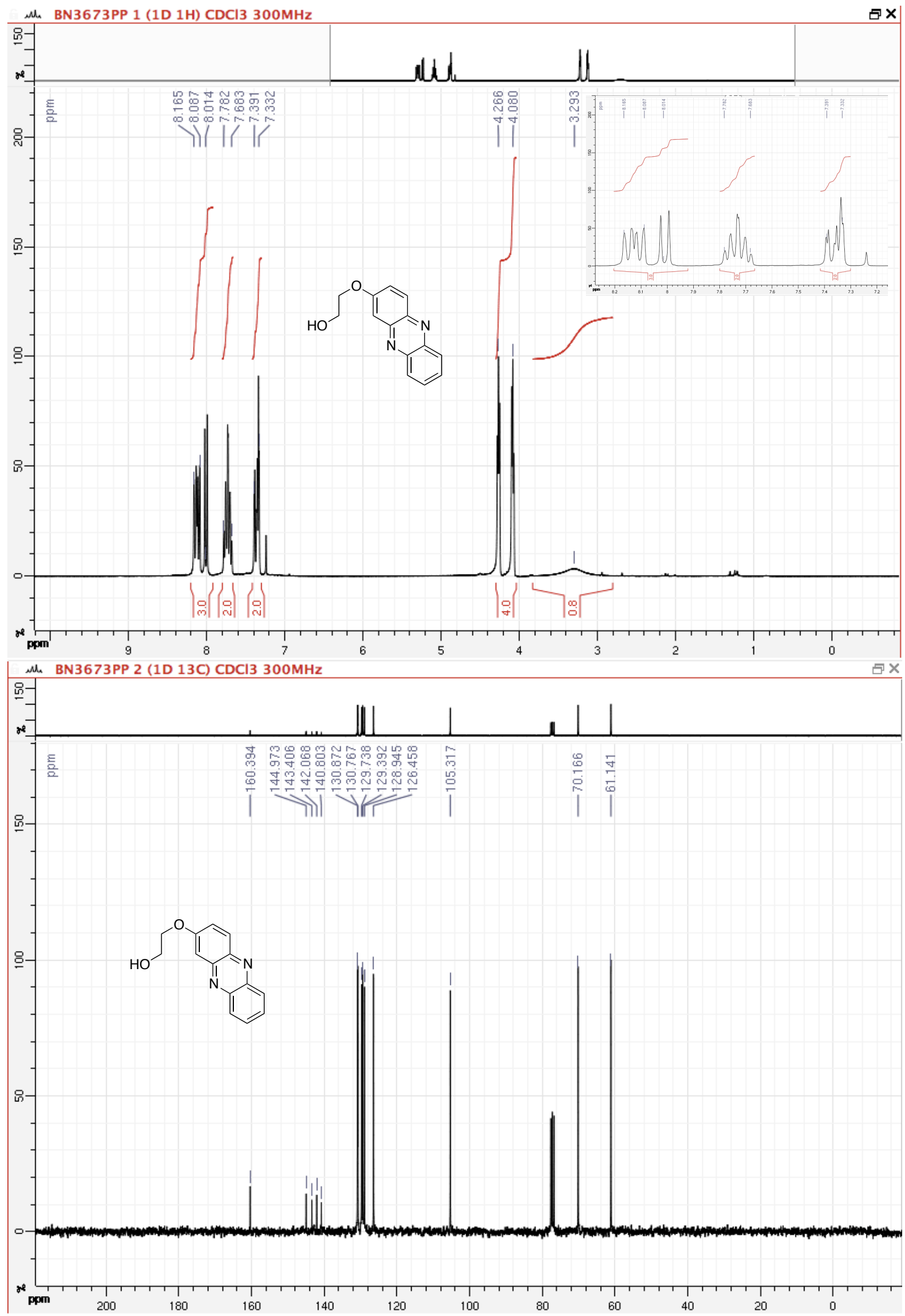

SI31 
Pyrido[2,3-a]phenazine (6)
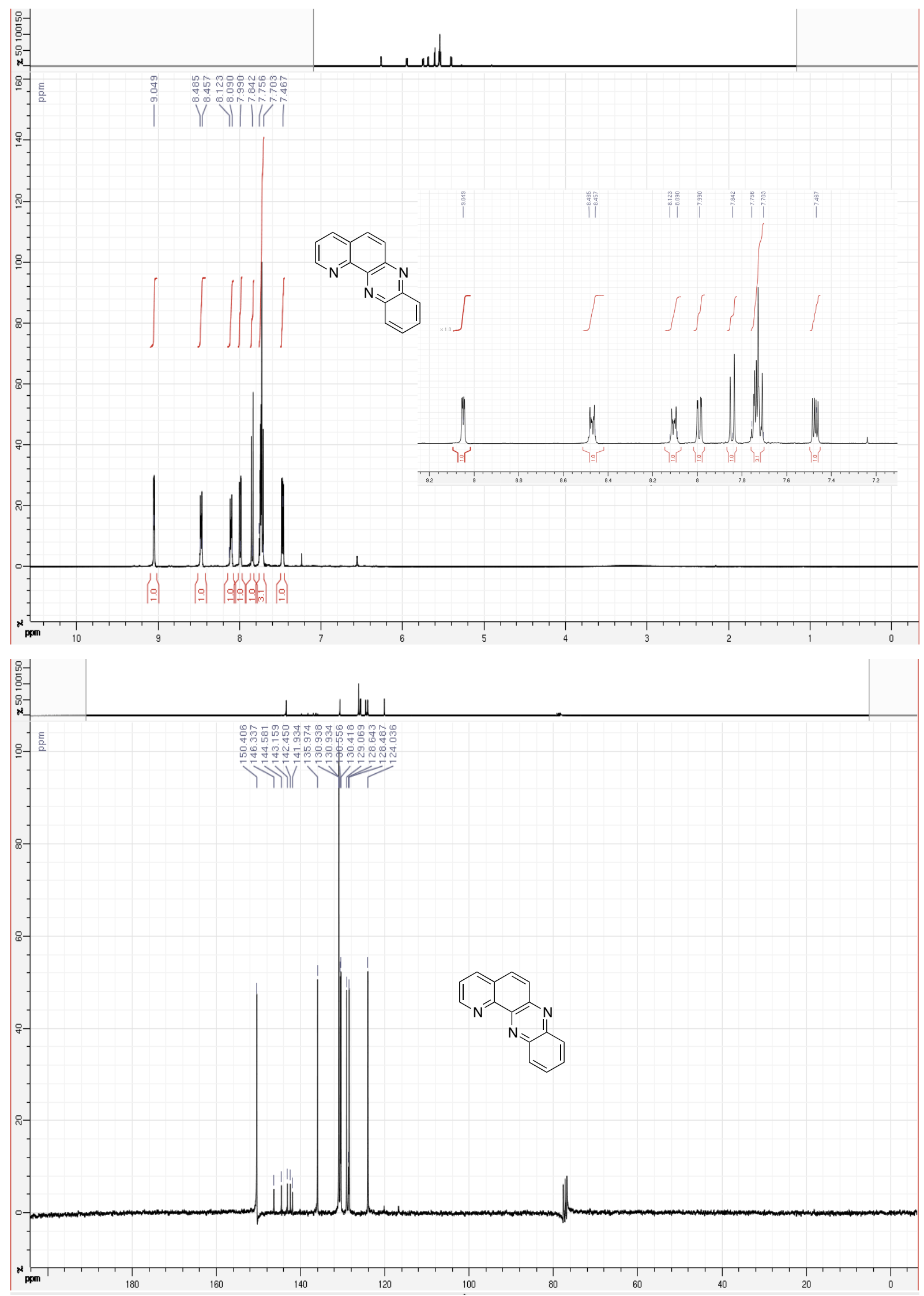

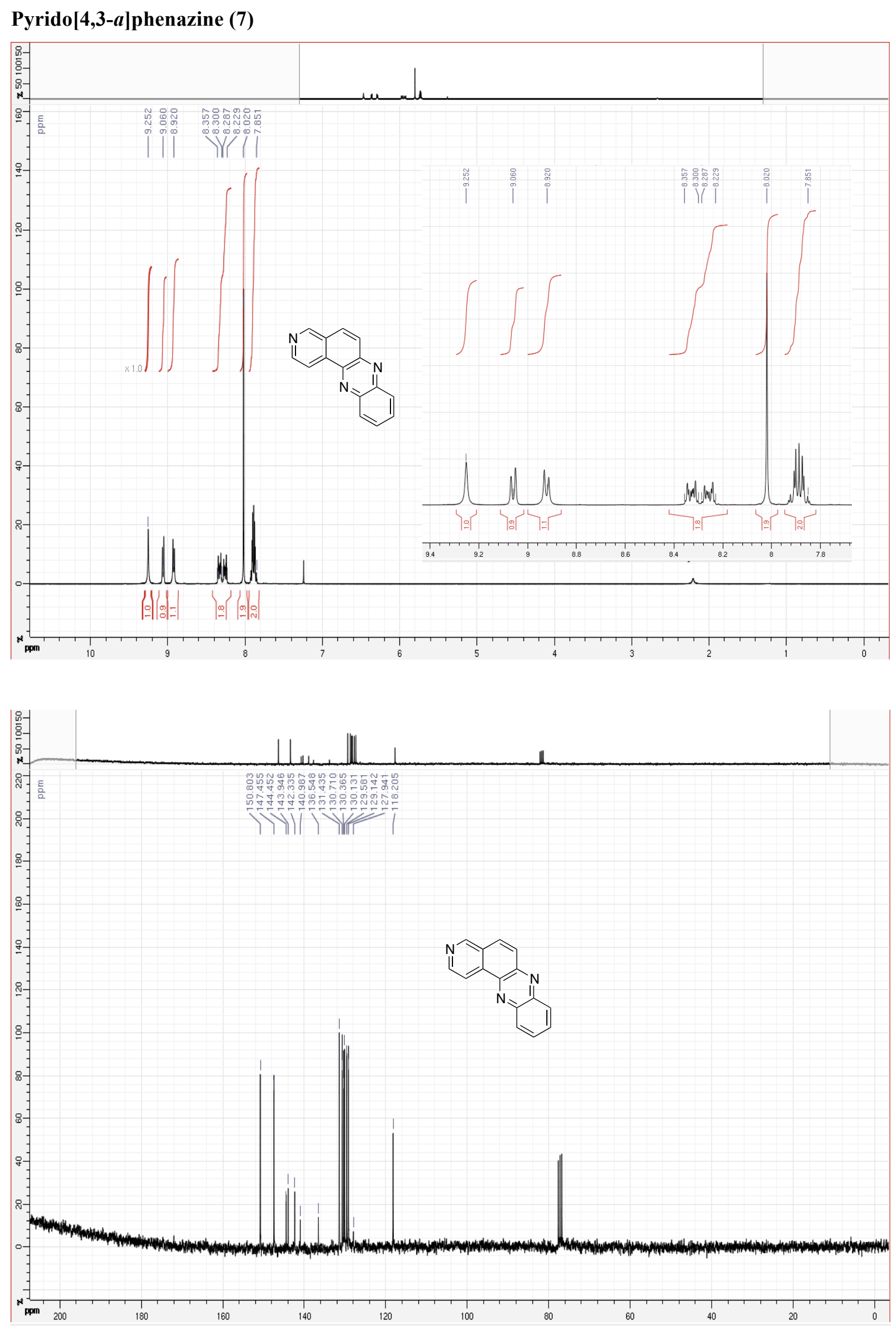